University of Nebraska - Lincoln

DigitalCommons@University of Nebraska - Lincoln

\title{
Variation in the fumonisin biosynthetic gene cluster in fumonisin- producing and nonproducing black aspergilli
}

\author{
Antonia Susca \\ Robert H. Proctor \\ USDA-ARS \\ Robert A. E. Butchko \\ USDA-ARS \\ Miriam Haidukowski \\ Institute of Sciences of Food Production \\ Gaetano Stea \\ Institute of Sciences of Food Production \\ See next page for additional authors
}

Institute of Sciences of Food Production, antonella.susca@ispa.cnr.it

Follow this and additional works at: https://digitalcommons.unl.edu/usdaarsfacpub

Susca, Antonia; Proctor, Robert H.; Butchko, Robert A. E.; Haidukowski, Miriam; Stea, Gaetano; Logrieco, Antonio; and Moretti, Antonio, "Variation in the fumonisin biosynthetic gene cluster in fumonisinproducing and nonproducing black aspergilli" (2014). Publications from USDA-ARS / UNL Faculty. 1569. https://digitalcommons.unl.edu/usdaarsfacpub/1569

This Article is brought to you for free and open access by the U.S. Department of Agriculture: Agricultural Research Service, Lincoln, Nebraska at DigitalCommons@University of Nebraska - Lincoln. It has been accepted for inclusion in Publications from USDA-ARS / UNL Faculty by an authorized administrator of DigitalCommons@University of Nebraska - Lincoln. 


\section{Authors}

Antonia Susca, Robert H. Proctor, Robert A. E. Butchko, Miriam Haidukowski, Gaetano Stea, Antonio Logrieco, and Antonio Moretti 


\title{
Variation in the fumonisin biosynthetic gene cluster in fumonisin-producing and nonproducing black aspergilli
}

\author{
Antonia Susca $^{\mathrm{a}, *, 1}$, Robert H. Proctor ${ }^{\mathrm{b}, 1}$, Robert A.E. Butchko ${ }^{\mathrm{b}}$, Miriam Haidukowski ${ }^{\mathrm{a}}$, Gaetano Stea ${ }^{\mathrm{a}}$, \\ Antonio Logrieco ${ }^{\text {a }}$, Antonio Moretti ${ }^{a}$ \\ ${ }^{a}$ Institute of Sciences of Food Production, CNR, via Amendola 122/0, 70126 Bari, Italy \\ ${ }^{\mathrm{b}}$ US Department of Agriculture, Agriculture Research Service, National Center for Agricultural Utilization Research, 1815 North University Street, Peoria, IL 61604-3902, USA
}

\section{A R T I C L E I N F O}

\section{Article history:}

Received 4 July 2014

Accepted 24 September 2014

Available online 2 October 2014

\section{Keywords:}

Black aspergilli

Grape

Fumonisin

Biosynthetic gene cluster

Gene cluster deletion

\begin{abstract}
A B S T R A C T
The ability to produce fumonisin mycotoxins varies among members of the black aspergilli. Previously, analyses of selected genes in the fumonisin biosynthetic gene (fum) cluster in black aspergilli from California grapes indicated that fumonisin-nonproducing isolates of Aspergillus welwitschiae lack six fum genes, but nonproducing isolates of Aspergillus niger do not. In the current study, analyses of black aspergilli from grapes from the Mediterranean Basin indicate that the genomic context of the fum cluster is the same in isolates of $A$. niger and A. welwitschiae regardless of fumonisin-production ability and that full-length clusters occur in producing isolates of both species and nonproducing isolates of $A$. niger. In contrast, the cluster has undergone an eight-gene deletion in fumonisin-nonproducing isolates of $A$. welwitschiae. Phylogenetic analyses suggest each species consists of a mixed population of fumonisinproducing and nonproducing individuals, and that existence of both production phenotypes may provide a selective advantage to these species. Differences in gene content of fum cluster homologues and phylogenetic relationships of fum genes suggest that the mutation(s) responsible for the nonproduction phenotype differs, and therefore arose independently, in the two species. Partial fum cluster homologues were also identified in genome sequences of four other black Aspergillus species. Gene content of these partial clusters and phylogenetic relationships of fum sequences indicate that non-random partial deletion of the cluster has occurred multiple times among the species. This in turn suggests that an intact cluster and fumonisin production were once more widespread among black aspergilli.
\end{abstract}

(c) 2014 Elsevier Inc. All rights reserved.

\section{Introduction}

The black aspergilli, also known as Aspergillus section Nigri (Gams et al., 1985), can cause food spoilage and occur worldwide on a wide variety of substrates, including soil, grains, forage crops, fruits, vegetables, nuts, cotton, and meat and dairy products (Raper and Fennell, 1965; Pitt and Hocking, 2007, 2009). Some black Aspergillus species are also important for commercial production of organic acids, hydrolytic enzymes, and fermented beverages (Hong et al., 2013; Raper and Fennell, 1965; Varga et al., 2000). Although multiple species of black aspergilli are difficult to distinguish from one another by morphology, differentiation between species has been facilitated by DNA sequence-based methods

\footnotetext{
* Corresponding author at: Institute of Sciences of Food Production, National Research Council (ISPA-CNR), via Amendola 122/0, 70126 Bari, Italy. Fax: +39 0805939374.

E-mail address: antonella.susca@ispa.cnr.it (A. Susca).

1 These authors contributed equally to this work.
}

(Hong et al., 2013; Perrone et al., 2011; Varga et al., 2011). For example, a recent DNA-based phylogenetic analysis resolved Aspergillus awamori from a collection of $A$. niger isolates (Perrone et al., 2011). A. awamori was renamed A. welwitschiae because it is distinct from black aspergilli used for fermentation of the Okinawan beverage awamori, and it is identical to a previously described black Aspergillus isolates from the plant Welwitschiae mirabilis (Hong et al., 2013).

Some black aspergilli can produce the mycotoxins ochratoxins (Abarca et al., 2003, 2004; Wicklow et al., 1996; Varga et al., 2000; Cabanes et al., 2002; Sage et al., 2004; Samson et al., 2004) and fumonisins (Frisvad et al., 2007, 2011; Perrone et al., 2011). Reports of these mycotoxins in raisins, grape must, and wine have raised food safety concerns about the occurrence of black aspergilli on grapes (Logrieco et al., 2009, 2010). Fumonisins are a health concern because they can cause multiple diseases in experimental rodents and some livestock, and they are epidemiologically associated with several human diseases, including oesophageal cancer (Marasas et al., 2004). Fumonisins were first identified in cultures 
of the fungus Fusarium $\sim 25$ years ago, and there is an extensive literature on production by different species as well as the genetics and biochemistry of fumonisin biosynthesis in Fusarium (Alexander et al., 2009; Rheeder et al., 1992; Uhlig et al., 2012). In contrast, fumonisin production in black aspergilli was first reported less than a decade ago (Frisvad et al., 2007), and as a result, the literature on production in Aspergillus is relatively small.

Fumonisins consist of a linear polyketide-derived backbone with two methyl, one amine, one to four hydroxyl, and two tricarboxyl ester groups located at various positions along the backbone. Genetic and biochemical analyses of Fusarium identified a 16 to 17gene fumonisin biosynthetic gene (abbreviated FUM in Fusarium, fum in Aspergillus) cluster that encodes biosynthetic enzymes necessary for formation of fumonisins as well as two transport proteins and a transcription factor (Alexander et al., 2009). A homologue of the Fusarium FUM cluster is present in the A. niger genome (Baker, 2006; Pel et al., 2007). The latter cluster includes homologues of 11 of the Fusarium genes: the polyketide synthase (fum1), hydroxylase (fum3, fum6, fum15), dehydrogenase (fum7), aminotransferase (fum8), acyl-CoA synthase (fum10), carbonyl reductase (fum13), condensation-domain protein (fum14), $\mathrm{ABC}$ transporter (fum19), and transcription factor (fum21) genes. The A. niger cluster also includes a short-chain dehydrogenase gene (designated here as sdr1) that is not present in the Fusarium cluster and whose function, if any, in fumonisin biosynthesis has not been reported (Baker, 2006; Pel et al., 2007). Notably absent from the $A$. niger fum cluster is a homologue of the Fusarium FUM2 gene, which is responsible for hydroxylation of the fumonisin backbone at carbon atom 10 (Proctor et al., 2006). The lack of a FUM2 homologue in the A. niger cluster is consistent with the results of a majority of studies showing that this species produces only fumonisins that lack a hydroxyl at carbon atom 10, e.g. fumonisins $B_{2}, B_{4}$, and $B_{6}$ $\left(\mathrm{FB}_{2}, \mathrm{FB}_{4}, \mathrm{FB}_{6}\right.$ respectively) (Frisvad et al., 2007, 2011; Mansson et al., 2010; Noonim et al., 2009).

With the recent taxonomic revision of the black aspergilli, fumonisin production has been confirmed in only two species: $A$. niger and A. welwitschiae (formerly A. awamori) (Perrone et al., 2011; Palumbo et al., 2013; Varga et al., 2010, 2011). However, the ability to produce fumonisins is not uniform among isolates of these two species. In six independent studies $28-81 \%$ of the $A$. niger and/or A. welwitschiae isolates examined produced fumonisins (Abrunhosa et al., 2011; Frisvad et al., 2011; Mogensen et al., 2010; Susca et al., 2010; Palumbo et al., 2011; Varga et al., 2010). This variation in ability begs the question: what is the genetic basis for the difference in fumonisin production and nonproduction in $A$. niger and $A$. welwitschiae? In one study that addressed this question in black aspergilli isolated from grapes grown in the Mediterranean Basin, the fum8 gene was not detected in most fumonisin-nonproducing isolates of $A$. niger that were examined. However, this study was done before DNA sequence analyses resolved $A$. niger into $A$. niger sensu stricto and A. welwitschiae (Perrone et al., 2011; Varga et al., 2011; Hong et al., 2013). Because both of these species can occur on grapes (Palumbo et al., 2013), it is possible that the collection of A. niger examined by Susca et al. (2010) included isolates of A. welwitschiae as well as A. niger. A subsequent study examined the presence of eight fum genes (fum1, fum3 [also designated as fum9], fum6 - fum8, fum13, fum14, and fum19) in A. niger and A. welwitschiae isolates from grapes grown in California (Palumbo et al., 2013). All eight genes were detected in $\mathrm{FB}_{2}$-nonproducing isolates of A. niger, but only fum 1 and fum 19 were detected in nonproducing isolates of $A$. welwitschiae. Thus, the results of two studies indicate that in $A$. niger and $A$. welwitschiae collectively, the absence of one or more fum genes could account for the $\mathrm{FB}_{2}$-nonproduction phenotype in some isolates but not others.

The objective of the current study was to clarify the gene content and organization of the fum cluster in $\mathrm{FB}_{2}$-nonproducing isolates of $A$. niger and $A$. welwitschiae recovered from grapes. PCR, Southern blot, and sequence data of the fum cluster region provide evidence that the fum cluster in nonproducing isolates of A. niger includes all 11 fum genes as well as sdr1, whereas the cluster in nonproducing isolates of $A$. welwitschiae has undergone a large deletion. These findings combined with the results of phylogenetic analyses suggest the mixtures of fumonisin-producing and nonproducing individuals arose independently in the two species. In addition, genome sequence data suggest that the fum cluster and perhaps fumonisin production were once more widespread among members of the black aspergilli than they currently are.

\section{Materials and methods}

\subsection{Fungal cultures}

In this study, we employ the species nomenclature for black aspergilli used by Hong et al. (2013). The 58 black Aspergillus isolates analyzed in this study were obtained from the agro-food important toxigenic fungi - ITEM (Istituto Tossine e Micotossine da Parassiti Vegetali) Culture Collection at the Institute of Sciences of Food Production (ISPA), National Research Council, Italy. Further information about the isolates (year of isolation, depositor, toxin production, etc.) are available at the ISPA Website (server.ispa.cnr.it/ITEM/Collection/). Fifty-one isolates were recovered from grapes grown in the Mediterranean Basin: nine from Greece (ITEM 5218, 5219, 5253, 5266, 5267, 5272, 5276, 5277, 5283), 15 from Italy (ITEM 4717, 4853, 4858, 4859, 4863, 6122, 6123, 6126, $6127,6128,7090,7091,7097,7468,10355), 16$ from Turkey (ITEM 11945, 12918, 14266, 14270, 14273, 14275, 14281, 14282, 14286 14297, 14303, 14305, 14307, 14308, 14309, 14310), three from Spain (ITEM 4947, 4951, 9720), six from Portugal (ITEM 4545, 4547, 4552, 6140, 6142, 6144), and two from Israel (ITEM 6292 and 6293). In addition, three isolates were recovered from cashew nuts, ITEM 11448 from Brazil and ITEM 11552 and ITEM 11553 from India; and two isolates were recovered from raisins, ITEM 10927 from Turkey and ITEM 10954 from Chile. The study also included the $A$. niger type strain ITEM 4501, which was recovered from a tannin-gallic acid fermentation in the USA, and the A. welwitschiae reference strain ITEM 4502 (Perrone et al., 2011), the origin of which is unknown.

\subsection{Fumonisin analysis}

All isolates were grown in darkness for 7 days on Czapek yeast autolysate medium with $20 \%$ sucrose (CY20S) solidified with agar according to the method published by Frisvad et al. (2007). Fumonisins were analyzed according to the method of Frisvad et al. (2007) with slight modifications (De Girolamo et al., 2011). One g of CY20S culture was extracted with $5 \mathrm{~mL}$ of methanol/water $(70: 30, v / v)$ on an orbital shaker for $60 \mathrm{~min}$. One hundred $\mu \mathrm{L}$ of the extract were diluted with $900 \mu \mathrm{L}$ of acetonitrile/water (30:70, $\mathrm{v} / \mathrm{v}$ ) and then filtered using RC $0.2 \mu \mathrm{m}$ filters (Phenomenex, U.S.A.). Fifty $\mu \mathrm{L}$ of diluted extract was derivatized by mixing with $50 \mu \mathrm{L}$ of o-phtaldialdehyde (OPA) for $50 \mathrm{~s}$. using a high performance liquid chromatograph (HPLC) autosampler (Agilent, Waldbronn, Germany). Three min. after the start of derivatization, $100 \mu \mathrm{L}$ of the extract was injected by full loop into an Agilent 1100 series HPLC (Agilent, Waldbronn, Germany). The analytical column was a Symmetry Shield RP18 $15 \mathrm{~cm} \times 4.6 \mathrm{~mm}, 5 \mu \mathrm{m}$ with a guard column inlet filter $(0.5 \mu \mathrm{m} \times 3 \mathrm{~mm}$ diameter, Rheodyne Inc. CA, USA), and the mobile phase consisting of a binary gradient was applied as follows: the initial composition of the mobile phase consisted of $57 \%$ solvent A (water-acetic acid, 99/1, v/v)/43\% solvent B (acetonitrile-acetic acid, 99/1, v/v), and was kept constant 
for $5 \mathrm{~min}$, then solvent B was linearly increased to $54 \%$ over $21 \mathrm{~min}$, then to $58 \%$ at $25 \mathrm{~min}$, after which the resulting solvent combination $(42 \% \mathrm{~A} / 58 \% \mathrm{~B})$ was held constant for $5 \mathrm{~min}$. The column temperature was $30^{\circ} \mathrm{C}$. The flow rate of the mobile phase was $0.8 \mathrm{~mL} / \mathrm{min}$. The excitation and emission wavelengths of the fluorometric detector were set at 335 and $440 \mathrm{~nm}$ respectively. Fumonisins were quantified by comparing chromatographic peak areas to those of standards. The detection limits for $\mathrm{FB}_{1}, \mathrm{FB}_{2}$ and $\mathrm{FB}_{3}$ were $0.1 \mu \mathrm{g} / \mathrm{g}$ based on a signal-to-noise ratio of $3: 1$.

\subsection{DNA isolation}

Fungal isolates were grown in liquid Wikerham's medium ( $40 \mathrm{~g}$ of glucose, $5 \mathrm{~g}$ of peptone, $3 \mathrm{~g}$ of yeast extract, $3 \mathrm{~g}$ of malt extract, and water up to $1 \mathrm{~L}$ ) on a rotary shaker $(120 \mathrm{rpm})$. Mycelia were harvested by filtration, lyophilized, and then ground using 5-mm iron beads in a Mixer Mill MM 400 (Retsch). Genomic DNA was extracted from $10 \mathrm{mg}$ of ground mycelia with the Wizard ${ }^{\circledR}$ Magnetic DNA Purification System for Food (Promega). The quality and quantity of the resulting DNA were assessed by agarose gel electrophoresis and by spectrometry with a Spectophotometer ND-1000 (Nano Drop).

\subsection{PCR amplifications}

PCR primers (Table 1) were used to screen for the presence of the fum cluster genes fum1, fum3, fum6, fum7, fum8, fum10, fum13, fum14, fum15, and fum21 (2 regions designated I and II), as well as the fum19-fum15 intergenic region and a region downstream of fum6. Primers were designed based on fum cluster sequences in A. niger strains ATCC 1015 (in the Joint Genome Institute (JGI) database) and CBS513.88 A. niger strains (in the GenBank database: accession AM269971.1) (Supplemental Table S1). When designing primers, we avoided regions where there was variability in the nucleotide sequences in A. niger strains ATCC 1015 and CBS513.88 whenever possible (Andersen et al., 2011). Primers CL1 and CL2A (O'Donnell et al., 2000) were used to amplify and sequence a $\sim 650$-bp fragment of the calmodulin gene (caM); primers BT2a and BT2b (Glass and Donaldson, 1995) were used to amplify sequence a 450 -bp fragment of the $\beta$-tubulin gene (ben $A$ ); and primers A-EF_F/A-EF_R (Perrone et al., 2011) were used to amplify and sequence a 700-bp fragment of the translation elongation factor $1 \alpha$ gene $(e f-1 \alpha)$.

Amplification reactions were performed in a $25-\mu \mathrm{L}$ volume: $0.6 \mathrm{U}$ of HotMaster Taq DNA Polymerase (5 Prime, Hamburg, Germany), 1x HotMaster Taq DNA Polymerase buffer with $25 \mathrm{mM} \mathrm{Mg}^{2+}, 300 \mathrm{mM}$ of each primer, $200 \mu \mathrm{M}$ of each deoxynucleoside triphosphate, and approximately $20 \mathrm{ng}$ of fungal DNA as template. Cycling conditions were as follows: denaturation at $94{ }^{\circ} \mathrm{C}$ for $2 \mathrm{~min}$; 35 cycles of denaturation at $94{ }^{\circ} \mathrm{C}$ for $50 \mathrm{~s}$, annealing at appropriate temperature for each primer pair for $30 \mathrm{~s}$, extension at $72{ }^{\circ} \mathrm{C}$ for $50 \mathrm{~s}$; final extension at $72{ }^{\circ} \mathrm{C}$ for $5 \mathrm{~min}$, followed by cooling at $4{ }^{\circ} \mathrm{C}$ until recovery of the samples. All PCR amplicons were examined by electrophoresis in a $1.2 \%(\mathrm{w} / \mathrm{v})$ ethidium bromide-stained agarose gel.

\subsection{DNA sequencing and phylogenetic analysis}

Prior to sequence analysis, amplicons were purified by the enzymatic mixture E. coli Exonuclease I/Shrimp Alkaline Phosphatase. The nucleotide sequence of both strands was determined with the Big-Dye Terminator Cycle Sequencing Ready Reaction Kit. Sequence reactions were purified by filtration through Sephadex G-50 (Pharmacia, Uppsala, Sweden) equilibrated in double-distilled water and then analyzed with a 3730 DNA Analyzer (Applied Biosystems). Sequence reads were viewed and when necessary edited with the BioNumerics 5.1 software (Applied Maths). To determine the nucleotide sequence of the fum cluster in A. niger strain ITEM 10355 and $A$. welwitschiae strains ITEM 7468 and ITEM 11945 , the entire genome sequence of each strain was obtained from BaseClear, a private company in Leiden, The Netherlands. Sequence data were generated by the Illumina Casava pipeline version 1.8 .2 sequencing system and included paired reads. The number of sequence reads was $22.3,12.3$ to 22.7 million and average

Table 1

PCR primer used for screen A. niger/A.welwitschiae strains.

\begin{tabular}{|c|c|c|c|c|c|c|}
\hline Primer name & Sequence & Tm primer & $\begin{array}{l}\text { Primer coordinates on FUM } \\
\text { CLUSTER-Scaffold } 1 \text { of } A \text {. niger } \\
\text { v1.0 (ATCC1015-JGI portal) }\end{array}$ & Amplicon size (bp) & Gene & Reference \\
\hline fum3r-234F & TACCATGGACCACTTTCCCG & 59 & $2,013,504-2,013,523$ & 651 & FUM3 & This work \\
\hline fum3-884R & AAGTTCCTCAAGCGGCAGTC & 58 & $2,014,135-2,014,154$ & & & \\
\hline fum13-200F & ATGCTCTTCACCTCCTCCGG & 60 & $2,008,576-2,008,595$ & 651 & FUM13 & This work \\
\hline fum13-850R & CACTCAACGAGGAGCCTTCG & 60 & $2,009,207-2,009,226$ & & & \\
\hline fum $14-102 F$ & TTGGGCTGATGTGCTCTGTC & 58 & $2,006,129-2,006,148$ & 730 & FUM14 & This work \\
\hline fum $14-831 R$ & ACCTCGTAGACGTAATTGAGTAGTCCT & 58 & $2,006,832-2,006,858$ & & & \\
\hline fum $10-756 \mathrm{~F}$ & GTCATTATTCCTCCGGCCCT & 59 & $2,016,981-2,017,000$ & 651 & FUM10 & This work \\
\hline fum10-1406 & TGGGATTCGAAAGCATACCG & 59 & $2,017,612-2,017,631$ & & & \\
\hline fum7-600F & CAACAGCCCGAATCCCAGTA & 60 & $2,015,113-2,015,132$ & 681 & FUM7 & This work \\
\hline fum7-1280R & GCTCAGTCTTGCCCATCGTG & 60 & $2,015,774-2,015,793$ & & & \\
\hline fum $1-4583 F$ & GGGTTCCAGGCAGAATCGTAC & 59 & $1,990,159-1,990,179$ & 701 & FUM1 & This work \\
\hline fum1-5283R & GAACTCACATCCTTTTGGGCC & 59 & $1,990,839-1,990,859$ & & & \\
\hline fum15-299F & CGATTGGTAGCCCGAGGAA & 60 & $1,999,811-1,999,829$ & 701 & FUM15 & This work \\
\hline fum15-999R & CTTGATATTGCGGAGTGGTCC & 58 & $2,000,491-2,000,511$ & & & \\
\hline fum15-14-1395F & CATTTCATGGGACCTCAGCC & 59 & $2,002,525-2,002,544$ & 703 & FUM21 I & This work \\
\hline fum15-14-2097R & AAGCACAGGTTCCGAATTTGA & 58 & $2,003,207-2,003,227$ & & & \\
\hline fum15-14-2997F & GGGTCCCATTGCCTCAATT & 58 & $2,004,127-2,004,145$ & 705 & FUM21 II & This work \\
\hline fum15-14-3701R & CAATGGAGTCGACGGTGTCAC & 60 & $2,004,811-2,004,831$ & & & \\
\hline fum19-15-62F & ACACCGCGAGAATTCCATG & 58 & $1,998,657-1,998,675$ & 868 & FUM19-FUM15 & This work \\
\hline fum19-15-929R & GCAGGCTGGTAGTAGCGACAT & 58 & $1,999,504-1,999,524$ & & & \\
\hline fum $>6-11 F$ & CAAAAGACACCGCCCGTCT & 60 & $2,023,641-2,023,659$ & 667 & Down-stream fum6 & This work \\
\hline fum $>6-677 R$ & TTGACGCCCTGTACAAGGC & 58 & $2,024,289-2,024,307$ & & & \\
\hline fum6-1701F & CTGTGAGGCCCTGGCACTT & 60 & $2,021,591-2,021,609$ & 849 & FUM6 & This work \\
\hline fum6-2549R & TCTGCCGGAGCTCAACGTA & 59 & $2,022,421-2,022,439$ & & & \\
\hline vnF1 & TTCGTTTGAGTGGTGGCA & 56 & $2,011,611-2,011,628$ & 862 & FUM8 & Susca et al. (2010) \\
\hline vnR3 & CAACTCCATASTTCWWGRRAGCCT & 57 & $2,010,766-2,010,789$ & & & \\
\hline
\end{tabular}


read length was 50, 99 and 98 for strains ITEM 10355, 7468 and 11945 respectively. Sequence reads were trimmed and assembled using the program CLC Genomics Workbench version 5.5.1 (CLCbio). The sequence coverage for the genomes was 32X for ITEM 10355 and ITEM 7468 and 60X for ITEM 11945. The fum cluster region was identified in the resulting assembled genome sequences with the Basic Local Alignment Search Tool (BLAST) (Altschul et al., 1990) function in CLC Genomics Workbench. Query sequences used in the BLAST analysis were from $A$. niger fum gene homologues recovered from the A. niger ATCC 1015 genome sequence database at the JGI website (http://genome.jgi-psf.org). Genes within the fum cluster region of the ITEM 10355, 7468 and 11945 genome sequences were then manually annotated.

Initial species identification of field isolates was determined by assessing the nucleotide sequence identity of caM amplicons with references sequences. BLAST (Altschul et al., 1990) at the National Center for Biotechnology Information (NCBI), caM sequences of the isolates were queried against NCBI accessions AJ964872 and AJ964874, the caM sequences of the $A$. niger type strain (ITEM $4501 \mathrm{~T}=\mathrm{CBS} 554.65 \mathrm{~T}$ ) and $A$. welwitschiae type strain (ITEM $4509 \mathrm{~T}=$ CBS 557.65T) respectively.

To infer phylogenetic relationships, nucleotide sequences of housekeeping and fum genes were aligned with ClustalW and then subjected to maximum likelihood and maximum parsimony analyses as implemented in MEGA5 (Tamura et al., 2011). All positions containing gaps and missing data were eliminated from the analyses. Statistical significance of branches in the resulting trees was assessed by bootstrap analysis using 500 or 1000 pseudoreplications (Felsenstein, 1985). Selection within orthologs of fum1 was assessed by estimating differences in rates of nonsynonymous and synonymous substitutions at each codon of fum1. This was accomplished using the program Selecton version 2.4, which estimates the ratio of nonsynonymous to synonymous substitution rates using a Bayesian approach (Stern et al., 2007).

\subsection{Southern blot analysis}

A subset of 11 Aspergillus isolates was examined for the presence of fum genes and sdr1 by Southern blot analysis. Genomic DNA was isolated from lyophilized mycelia that was ground to a powder and then suspended in extraction buffer (200 mM Tris$\mathrm{Cl}, \mathrm{pH} 8,250 \mathrm{mM} \mathrm{NaCl}, 25 \mathrm{mM}$ EDTA, pH 8, and 0.5\% SDS) at $50 \mathrm{mg}$ per $250 \mu \mathrm{L}$ of buffer. Genomic DNA was purified from the suspension with the DNeasy Plant Mini Kit (Qiagen) following the protocol recommended by the manufacturer. Approximately $5 \mu \mathrm{g}$ of genomic DNA was digested with restriction enzyme HindIII, electrophoresed on a $1 \%$ agarose gel, transferred to a nylon membrane, and then subjected to a standard Southern hybridization protocol (Sambrook et al., 1989). Southern hybridization probes were generated by standard PCR with either the same primers used for the PCR screen (Table 2) or primers that amplified a fragment that spanned but was larger than the amplicon from the PCR screen. Following amplification, DNA fragments were subjected to agarose gel electrophoresis, purified with the UltraClean (MoBio Laboratories) method, and then labelled with ${ }^{32} \mathrm{P}$ using the Readyto-Go DNA labelling kit (Amersham Biosciences).

\section{Results}

\subsection{Species identification}

Initial species identities of 56 Aspergillus isolates employed in this study were determined by comparisons of the nucleotide sequence of the caM sequence from each isolate with reference caM sequences from the type strains of $A$. niger (ITEM 4501) and
A. welwitschiae (ITEM 4509). The caM sequences from 19 isolates were $>99 \%$ identical to that of type strain ITEM 4501 , indicating that they were $A$. niger. The caM sequences from 34 other isolates were $>99 \%$ identical to the caM sequence of type strain 4509 , indicating that they were $A$. welwitschiae. The identities of three other isolates, ITEM 11448, ITEM 11552 and ITEM 11553, were ambiguous because their caM sequences exhibited $99.0 \%$ and $98.8 \%$ identity to the reference sequences for $A$. niger and $A$. welwitschiae respectively. These three isolates differ from all other isolates examined in that they were isolated from dried fruit or cashew nuts from Brazil (ITEM 11448) or India (ITEM 11552 and ITEM 11553) rather than from grapes or raisins in the Mediterranean Basin.

To further resolve relationships of the Aspergillus strains, nucleotide sequences of fragments of three genes, ben $A$, caM and ef- $1 \alpha$, from a subset of 51 isolates were subjected to maximum likelihood (ML) and maximum parsimony (MP) analysis. The caM sequences resolved isolates identified as either $A$. niger or $A$. welwitschiae into separate well-supported clades in the ML and MP analyses, but analyses with either benA or ef- $1 \alpha$ sequences did not consistently resolve isolates of these two species into monophyletic clades (Supplemental Fig. S1). Because there were no conflicting branches that were strongly supported in trees generated with individual genes, we conducted ML and MP analyses with the concatenated ben $A, c a M$ and $e f-1 \alpha$ sequences. These analyses resolved isolates of the two species into separate and well-supported clades (Fig. 1). However, bootstrap values (84-96) for the clades corresponding to each species were not as high as obtained with the caM sequence alone. Isolates ITEM 11552 and ITEM 11553, which had ambiguous identities based on caM sequence comparisons, were resolved as a distinct well-supported clade that was basal to the A. welwitschiae clade (Fig. 1). The analyses did not provide strong support for the placement of isolate ITEM 11448. Thus, the phylogenetic analyses did not provide definitive species identities for isolates ITEM 11448, ITEM 11552 and ITEM 11553.

\subsection{Fumonisins production}

Because $A$. niger produces $\mathrm{FB}_{2}$ at levels that are 8 and 200 times as high as $\mathrm{FB}_{4}$ and $\mathrm{FB}_{6}$ respectively (Frisvad et al., 2011), we employed $\mathrm{FB}_{2}$ as a marker for fumonisin production. To assess $\mathrm{FB}_{2}$ production, we grew isolates on CY20S, a medium that supports some of the highest levels of fumonisin production reported for A. niger (Frisvad et al., 2007). HPLC analysis of CY20S cultures of 58 Aspergillus isolates revealed that $14 \mathrm{~A}$. niger isolates, including the $A$. niger type strains ITEM 4501, produced $0.5-3.3 \mu \mathrm{gB}_{2}$ per g culture, and $10 \mathrm{~A}$. welwitschiae isolates, including the $A$. welwitschiae reference strains ITEM 4502, produced 1.0-25.3 $\mu \mathrm{g} \mathrm{FB}_{2}$ per $\mathrm{g}$ culture. Six A. niger strains and $25 \mathrm{~A}$. welwitschiae strains did not produce detectable levels of $\mathrm{FB}_{2}$ under the conditions examined, nor did strains ITEM 11448, ITEM 11552 and ITEM 11553. The analytical method employed in this study was able to detect $\mathrm{FB}_{1}$ and $\mathrm{FB}_{3}$ in addition to $\mathrm{FB}_{2}$. However, neither $\mathrm{FB}_{1}$ nor $\mathrm{FB}_{3}$ was detected in any of $A$. niger or $A$. welwitschiae cultures examined.

In order to gain insight into the relationships of $\mathrm{FB}_{2}$-producing and nonproducing isolates of Aspergillus, we examined the production phenotypes in the context of the phylogeny generated with the concatenated sequences of three housekeeping genes: ben $A$, $c a M$ and $e f-1 \alpha$. Although phylogenetic analysis resolved $A$. welwitschiae and $A$. niger into separate well-supported monophyletic clades, the analysis did not provide strong support for multiple clades within either species. Within A. welwitschiae, however, there was one well-supported sub-specific clade (ML bootstrap value $=85$ ) that consisted of the majority of $A$. welwitschiae isolates analyzed, including both $\mathrm{FB}_{2}$-producing and nonproducing isolates (Fig. 1). This sub-specific clade provides evidence that some 
Table 2

PCR screening of $A$. niger and $A$. welwitschiae strains versus fum genes and intergenic regions.

\begin{tabular}{|c|c|c|c|c|c|c|c|c|c|c|c|c|c|c|c|}
\hline ITEM & $\begin{array}{l}\text { Aspergillus } \\
\text { species }\end{array}$ & $\begin{array}{c}{ }^{*} \mathbf{F B}_{2} \\
\text { Production }\end{array}$ & $\begin{array}{c}\text { fum1 } \\
(701 \mathrm{bp})\end{array}$ & $\begin{array}{l}\text { fum 19-15 } \\
\text { (868 bp) }\end{array}$ & $\begin{array}{c}\text { fum15 } \\
\text { (701 bp) }\end{array}$ & $\begin{array}{l}\text { fum21 I } \\
(703 \mathrm{bp})\end{array}$ & $\begin{array}{l}\text { fum21 II } \\
(705 \mathrm{bp})\end{array}$ & $\begin{array}{c}\text { fum14 } \\
(730 \mathrm{bp})\end{array}$ & $\begin{array}{c}f u m 13 \\
\text { (651 bp) }\end{array}$ & $\begin{array}{c}\text { fum } 8 \\
(800 \mathrm{bp})\end{array}$ & $\begin{array}{c}\text { fum } 3 \\
(651 \mathrm{bp})\end{array}$ & $\begin{array}{c}\text { fum } 7 \\
\text { (681 bp) }\end{array}$ & $\begin{array}{c}\text { fum10 } \\
\text { (651 bp) }\end{array}$ & $\begin{array}{c}\text { fum6 } \\
\text { (849 bp) }\end{array}$ & $\begin{array}{c}\text { Dowstream } \\
\text { fum6 } \\
\text { (667 bp) }\end{array}$ \\
\hline 5266 & A. welwitschiae & + & + & + & + & + & + & + & + & + & + & + & + & + & + \\
\hline 7097 & A. welwitschiae & + & +1 & + & +1 & + & +1 & +1 & +1 & +1 & +1 & +1 & +1 & +1 & + \\
\hline 14303 & A. welwitschiae & + & $\begin{array}{l}+ \\
+\end{array}$ & + & + & $\begin{array}{llll}+ & & l & l\end{array}$ & + & + & + & $\begin{array}{llll}+ & & & \end{array}$ & $\begin{array}{lll}+ & & \end{array}$ & $\begin{array}{llll}+ & & & \end{array}$ & + & $\begin{array}{llll}+ & & & \end{array}$ & + \\
\hline 14305 & A. welwitschiae & + & + & + & $\begin{array}{l}+ \\
+\end{array}$ & + & $\begin{array}{lll}+ & & \end{array}$ & + & + & + & 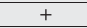 & + & + & 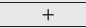 & + \\
\hline 14308 & A. welwitschiae & + & + & + & 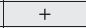 & + & + & + & + & + & 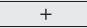 & + & 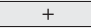 & $\begin{array}{lll}+ & & \end{array}$ & + \\
\hline 11945 & A. welwitschiae & + & + & + & + & + & + & + & + & + & $\begin{array}{l}+ \\
+\end{array}$ & + & + & + & + \\
\hline 14297 & A. welwitschiae & + & + & + & + & + & + & + & + & + & + & + & + & + & + \\
\hline 4502 & A. welwitschiae & + & +1 & +1 & +1 & +1 & +1 & +1 & +1 & +1 & +1 & +1 & +1 & +1 & + \\
\hline 5277 & A. welwitschiae & + & + & + & + & n.t. & + & + & + & + & + & + & + & + & n.t \\
\hline 7468 & A. welwitschiae & - & + & + & + & - & - & - & - & - & - & - & - & - & + \\
\hline 5253 & A. welwitschiae & - & + & + & + & - & - & 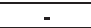 & 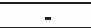 & 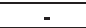 & 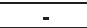 & 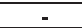 & - & 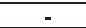 & + \\
\hline 6122 & A. welwitschiae & - & + & + & + & - & - & - & - & - & - & - & - & - & + \\
\hline 6127 & A. welwitschiae & - & $t^{1}$ & + & $+{ }^{1}$ & - & -1 & -1 & -1 & -1 & -1 & -1 & -1 & -1 & + \\
\hline 6293 & A. welwitschiae & - & $+{ }^{1}$ & + & $+{ }^{1}$ & - & -1 & -1 & -1 & -1 & -1 & -1 & -1 & -1 & + \\
\hline 14310 & A. welwitschiae & - & +1 & + & $+{ }^{1}$ & - & $-{ }^{1}$ & -1 & -1 & -1 & $-{ }^{1}$ & -1 & -1 & $-{ }^{1}$ & + \\
\hline 4552 & A. welwitschiae & 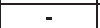 & + & + & + & - & - & - & - & - & - & - & 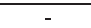 & - & n.t \\
\hline 4545 & A. welwitschiae & - & + & + & + & - & - & - & - & - & - & - & - & - & + \\
\hline 4717 & A. welwitschiae & 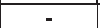 & + & + & + & - & - & - & - & - & - & - & - & - & + \\
\hline 4859 & A. welwitschiae & - & + & + & + & - & - & - & - & - & - & - & - & - & + \\
\hline 4863 & A. welwitschiae & - & + & + & + & - & - & - & - & - & - & - & - & - & + \\
\hline 4947 & A. welwitschiae & - & + & + & + & - & - & - & - & - & - & - & - & - & + \\
\hline 5267 & A. welwitschiae & 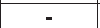 & + & + & + & - & 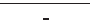 & - & - & - & - & 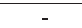 & $\begin{array}{lll}- \\
-\end{array}$ & - & + \\
\hline 5272 & A. welwitschiae & - & + & + & + & - & - & - & - & - & - & - & - & - & + \\
\hline 5283 & A. welwitschiae & 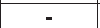 & + & + & + & n.t. & n.t. & 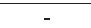 & 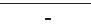 & 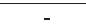 & - & 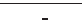 & $\begin{array}{lll}- \\
-\end{array}$ & n.t & n.t \\
\hline 6123 & A. welwitschiae & - & + & + & + & n.t. & n.t. & - & - & - & - & - & - & n.t & n.t \\
\hline 6126 & A. welwitschiae & $\begin{array}{ll}- \\
-\end{array}$ & + & + & + & - & - & 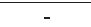 & - & - & - & 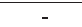 & 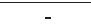 & - & n.t \\
\hline 6128 & A. welwitschiae & 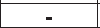 & + & + & + & - & - & - & - & - & - & 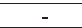 & - & n.t & n.t \\
\hline 6140 & A. welwitschiae & $\begin{array}{lll}- \\
-\end{array}$ & + & + & + & - & - & - & - & - & - & 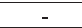 & - & n.t & n.t \\
\hline 6142 & A. welwitschiae & - & + & n.t. & n.t. & - & - & - & - & - & - & - & - & n.t & n.t \\
\hline 6144 & A. welwitschiae & 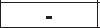 & + & + & + & - & - & - & - & - & - & - & - & - & n.t \\
\hline 14307 & A. welwitschiae & - & + & + & + & - & - & - & - & - & - & - & - & n.t & n.t \\
\hline 14309 & A. welwitschiae & $\begin{array}{lll}- & & \end{array}$ & + & - & + & n.t. & n.t. & - & - & - & - & - & - & n.t & n.t \\
\hline 11448 & Aspergillus sp. & - & + & + & + & - & - & - & - & - & - & - & - & $\overline{-}$ & + \\
\hline 11552 & Aspergillus sp. & 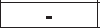 & $\begin{array}{llll}+ & & l & 0\end{array}$ & + & 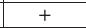 & - & - & - & - & 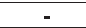 & - & 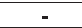 & - & 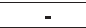 & + \\
\hline 11553 & Aspergillus sp. & - & + & + & + & - & - & - & - & - & - & - & - & - & + \\
\hline $4501^{T}$ & A. niger & + & +1 & + & +1 & + & +1 & +1 & +1 & +1 & +1 & +1 & +1 & +1 & + \\
\hline 7091 & A. niger & + & + & + & + & + & + & + & + & + & + & + & + & + & + \\
\hline 5276 & A. niger & + & + & + & + & + & + & + & + & + & + & + & + & + & + \\
\hline 4547 & A. niger & + & +1 & + & $+{ }^{1}$ & + & +1 & $+{ }^{1}$ & $+{ }^{1}$ & +1 & $+{ }^{1}$ & $+{ }^{1}$ & +1 & +1 & + \\
\hline 10927 & A. niger & + & + & + & + & + & + & + & + & + & + & + & + & + & + \\
\hline 14270 & A. niger & + & + & + & + & n.t. & + & + & + & + & + & + & + & n.t & n.t \\
\hline 14273 & A. niger & + & + & + & + & n.t. & + & + & + & + & + & + & + & n.t & n.t \\
\hline 14275 & A. niger & + & + & + & + & n.t. & + & + & + & + & + & + & + & n.t & n.t \\
\hline 14281 & A. niger & 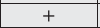 & + & + & + & n.t. & + & + & + & + & + & + & + & n.t & n.t \\
\hline 14282 & A. niger & + & + & + & + & n.t. & + & + & + & + & + & + & + & n.t & n.t \\
\hline 5218 & A. niger & - & + & + & + & + & + & + & + & + & + & + & + & + & + \\
\hline 5219 & A. niger & - & $t^{1}$ & + & $+^{1}$ & + & $t^{1}$ & +1 & $t^{1}$ & +1 & $t^{1}$ & $+{ }^{1}$ & $+^{1}$ & $t^{1}$ & + \\
\hline 7090 & A. niger & - & + & + & + & + & + & + & + & + & + & + & + & + & + \\
\hline 12918 & A. niger & - & + & + & + & + & + & + & + & + & + & + & + & + & + \\
\hline 9720 & A. niger & - & + & + & + & + & + & + & + & + & + & + & + & + & + \\
\hline 10355 & A. niger & - & + & + & + & + & + & + & + & + & + & + & + & + & + \\
\hline 10954 & A. niger & - & + & n.t & n.t & + & + & + & + & n.t & + & + & + & + & + \\
\hline
\end{tabular}

Bold character: Strains with all amplicons sequenced.

n.t. $=$ Not tested

${ }^{1}$ Data confirmed by Southern blot analysis.

${ }^{*} \operatorname{LOD}\left(\mathrm{FB}_{2}\right)=0.1 \mu \mathrm{g} / \mathrm{g}$.

${ }^{\mathrm{T}}$ Type strain.

$\mathrm{FB}_{2}$-nonproducing isolates of $A$. welwitschiae are more closely related to producing isolates than they are to other nonproducing isolates. Resolution of sub-specific clades within $A$. niger was not sufficient to draw conclusions about the relatedness of producing and nonproducing isolates in this species.

\section{3. fum Cluster analysis}

All experimental isolates were screened by PCR to assess the presence and/or absence of the fum cluster. The PCR assay employed primer pairs designed to amplify 13 different regions within the cluster (Fig. 2). Eleven fragments corresponded to segments within the coding regions of fum1, fum15, fum 21 (two segments designated I and II), fum14, fum13, fum8, fum3, fum7, fum10 and fum6; one fragment corresponded to the fum19-fum15 intergenic region; and one fragment corresponded to sequence downstream of the fum6 coding region (Fig. 2). Fragments of the expected size were amplified for all 13 regions from $\mathrm{FB}_{2}$-producing isolates of $A$. niger and $A$. welwitschiae as well as from all $\mathrm{FB}_{2}$-nonproducing isolates of $A$. niger (Table 2 and Fig. 2). In contrast, only fragments corresponding to fum1, fum15, the fum19-fum15 intergenic region, and the region downstream of fum 6 were amplified from $\mathrm{FB}_{2}$-nonproducing isolates of $A$. welwitschiae and isolates ITEM 11448, ITEM 11552 and ITEM 11553 (Table 2).

Southern blot analysis was done to obtain additional evidence for the presence and absence of fum genes in a subset of 11 isolates. This analysis employed hybridization probes for all 12 genes in the A. niger fum cluster: fum1, fum19, fum15, fum21, fum14, fum13, fum8, fum3, fum7, fum10, fum6, and sdr1. The results of the 


\section{benA, caM, ef-1 $\alpha$ 1761 characters}

\begin{tabular}{|l|}
\hline Portugal \\
\hline Spain \\
\hline Italy \\
\hline Greece \\
\hline Turkey \\
\hline India \\
Cheile \\
\hline Brazil \\
\hline
\end{tabular}
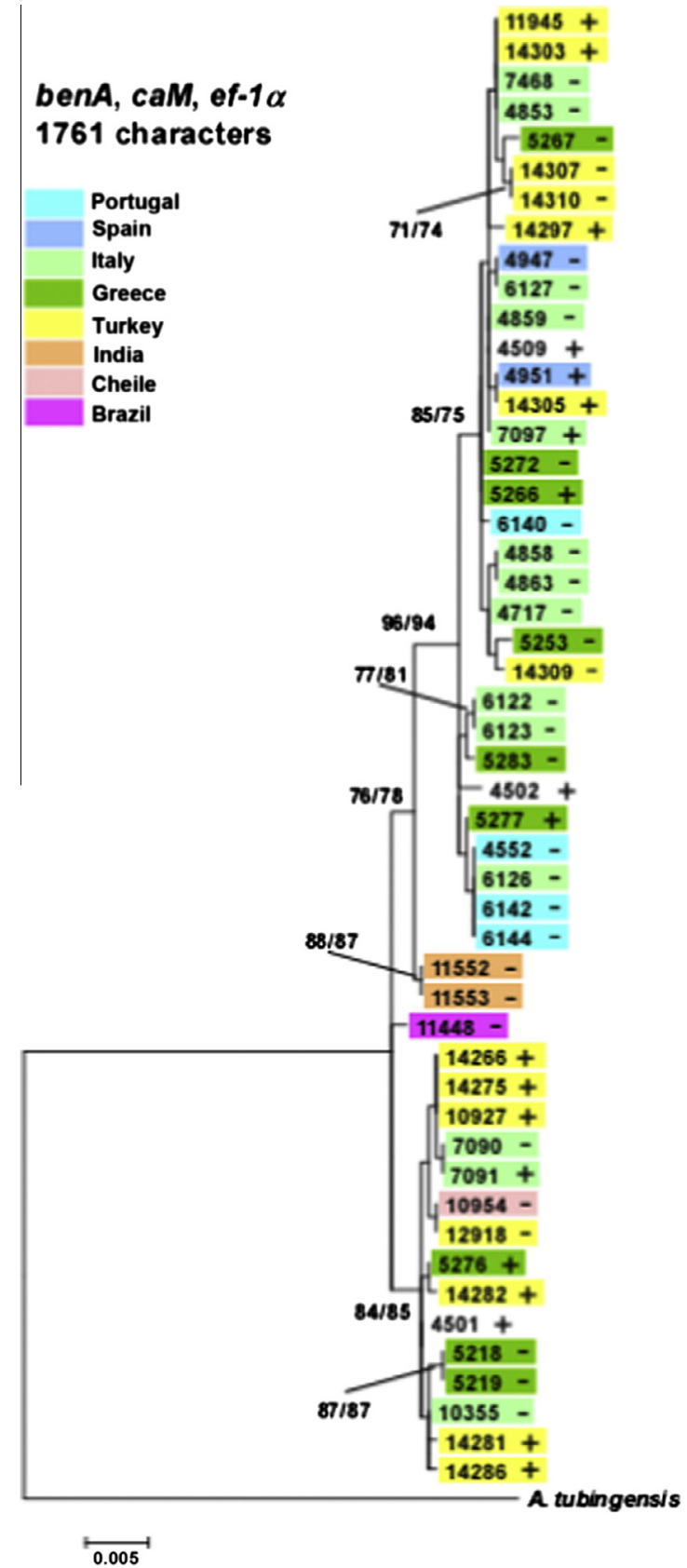

\section{A. welwitschiae}

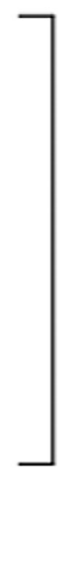

\section{A. niger}

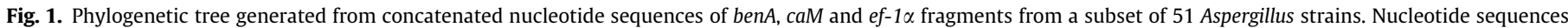

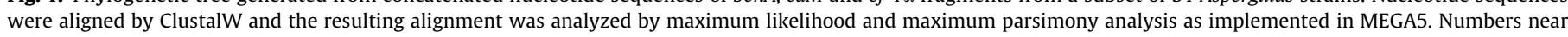

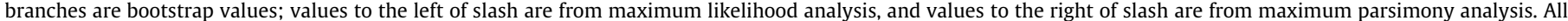

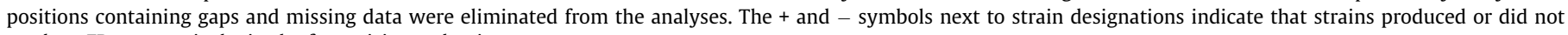
produce $\mathrm{FB}_{2}$, respectively, in the fumonisin production assay.

Southern analysis were consistent with the PCR data; hybridization signals were detected for all 12 genes in $\mathrm{FB}_{2}$-producing isolates of A. niger and $A$. welwitschiae as well as for nonproducing isolates of A. niger, but hybridization signals were detected for only fum 1 , fum19 and fum15 in $\mathrm{FB}_{2}$-nonproducing isolates of $A$. welwitschiae (Table 3; Supplemental Fig. S2).

Nucleotide sequence data for the entire fum cluster in two $\mathrm{FB}_{2}$ producing strains (CBS 513.88 and ATCC 1015) of A. niger have been reported previously (Pel et al., 2007; Baker, 2006). To further assess nucleotide sequence, content and arrangement of genes in the fum cluster among isolates of Aspergillus, we obtained sequence data for the fum region in $\mathrm{FB}_{2}$-nonproducing isolates of $A$. niger (ITEM 10355) and A. welwitschiae (ITEM 7468) as well as in an
$\mathrm{FB}_{2}$-producing isolate of $A$. welwitschiae (ITEM 11945). In A. niger, the content and arrangement of fum cluster genes is the same for the $\mathrm{FB}_{2}$-producing and nonproducing isolates examined (Fig. 3). The sequence data also indicate that the fum genes in the $\mathrm{FB}_{2}$-nonproducing isolate (ITEM 10355) of $A$. niger are functional; i.e. the genes do not include insertions or deletions that would introduce frame shifts or premature stop codons relative to the orthologues in the two producing strains. However, the fum 1 coding region in the nonproducing isolate of $A$. niger includes a 12-nucleotide deletion that is not present in the fum 1 homologues of producing strains CBS 513.88 and ATCC 1015. The fum1-encoded polyketide synthase catalyzes synthesis of the fumonisin backbone (Alexander et al., 2009; Du et al., 2008), therefore mutations that 

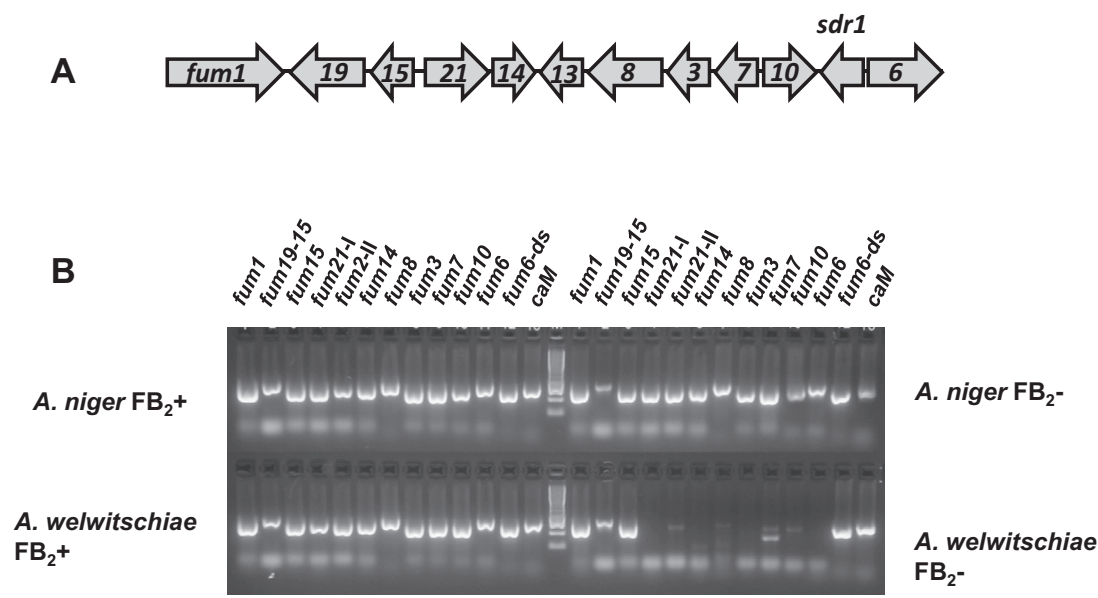

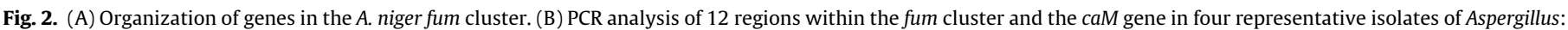

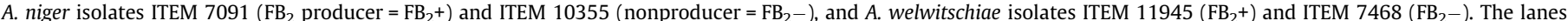

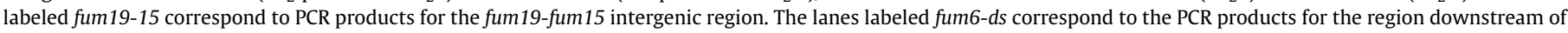

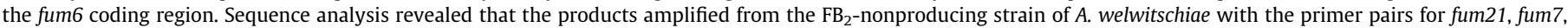
fum 8 and fum 10 were nonspecific amplicons.

Table 3

Southern blot analysis of fum genes ${ }^{\mathrm{a}}$ in selected strains of $A$. niger and A. welwitschiae.

\begin{tabular}{|c|c|c|c|c|c|c|c|c|c|c|c|c|c|}
\hline Species & ITEM No. & fum1 & fum19 & fum 15 & fum 21 & fum14 & fum13 & fum8 & fum3 & fum7 & fum 10 & sdr1 & fum6 \\
\hline A. niger & 4501 & + & + & + & + & + & + & + & + & + & + & + & + \\
\hline A. niger & 4547 & + & + & + & + & + & + & + & + & + & + & + & + \\
\hline A. niger & $5219^{b}$ & + & + & + & + & + & + & + & + & + & + & + & + \\
\hline A. niger & 6292 & + & + & + & + & + & + & + & + & + & + & + & + \\
\hline A. welwitschiae & 4502 & + & + & + & + & + & + & + & + & + & + & + & + \\
\hline A. welwitschiae & $4858^{\mathrm{b}}$ & + & + & + & - & - & - & - & - & - & - & - & - \\
\hline A. welwitschiae & $6127^{\mathrm{b}}$ & + & + & + & - & - & - & - & - & - & - & - & - \\
\hline A. welwitschiae & $6293^{b}$ & + & + & + & - & - & - & - & - & - & - & - & - \\
\hline A. welwitschiae & 7097 & + & + & + & + & + & + & + & + & + & + & + & + \\
\hline A. welwitschiae & $14310^{\mathrm{b}}$ & + & + & + & - & - & - & - & - & - & - & - & - \\
\hline
\end{tabular}

a The + and - symbols indicate that a signal was or was not detected, respectively, in the Southern analysis of fum genes and sdr1.

b Strains that did not produce fumonisins under the experimental conditions.

render the gene nonfunctional would result in a nonproduction phenotype. We used PCR and sequence analysis to determine whether the 12-nucleotide deletion was present in five other nonproducing and three producing isolates of $A$. niger. The deletion was not present in any of these isolates. Thus, if the deletion is responsible for fumonisin nonproduction in isolate ITEM 10355 , it is not responsible for nonproduction in some other nonproducing isolates of $A$. niger.

In an attempt to identify other mutations that could be responsible for the $\mathrm{FB}_{2}$-nonproduction phenotype in A. niger, we utilized fum-gene fragments amplified in the PCR screen (Table 2) from a subset of six $\mathrm{FB}_{2}$-producing and six nonproducing isolates of $A$. niger. Sequence analysis revealed that, with one exception, fragments from nonproducing isolates did not include nucleotide substitutions, insertions or deletions that would introduce premature stop codons into fum-gene coding sequences. The exception was a substitution that introduced a premature stop codon into the fum10 coding region of nonproducing isolate ITEM 12918. Functional analysis of the $F$. verticillioides fum 10 homologue indicates that it is required for formation of $\mathrm{FB}_{2}$ (Butchko et al., 2006; Zaleta-Rivera et al., 2006). Thus, the premature stop codon fum10 could account for the lack of $\mathrm{FB}_{2}$ production in ITEM 12918. Although we did not observe nucleotide changes that would have an obvious effect on fum gene function in other nonproducing isolates, it should be kept in mind that the sequences of the PCR products represented only $\sim 30 \%$ of coding regions in the fum cluster, and mutations could exist in gene segments that were not sequenced.

The content and arrangement of genes in the fum cluster of the $\mathrm{FB}_{2}$-producing isolate (ITEM 11945) of $A$. welwitschiae was the same as in the $A$. niger isolates examined. That is, the ITEM 11945 cluster includes homologues of the 11 known fum genes as well as sdr1 (Fig. 3). In contrast, the fum cluster in the $\mathrm{FB}_{2}$-nonproducing isolate of $A$. welwitschiae (ITEM 7468) includes full-length homologues of fum1, fum 19 and fum 15 as well as a truncated homologue of fum 6 but no other fum genes (Fig. 3); there is no evidence for fum21, fum14, fum13, fum8, fum3, fum7, fum10, and sdr1 in this region or elsewhere in the genome of ITEM 7468. The sequence data are consistent with the PCR and Southern analyses described above (Tables 2 and 3). Failure to detect a truncated fum6 in nonproducing isolates of A. welwitschiae in the PCR and Southern analyses can be explained by the fact that the probe used in each analysis did not correspond to the region of fum6 present in ITEM 7468.

\subsection{Partial fum cluster in black aspergilli}

Analysis of genome sequence databases available at the JGI and NCBI revealed the presence of remnants of fum cluster homologues in three other black aspergilli species: A. brasiliensis, A. tubingensis, and A. luchuensis strains CBS 106.47 (synonym A. acidus) and IFO 4308 (synonym A. kawachii, Futagami et al., 2011), (Supplemental 


\title{
A. niger ATCC 1015 FB producer
}

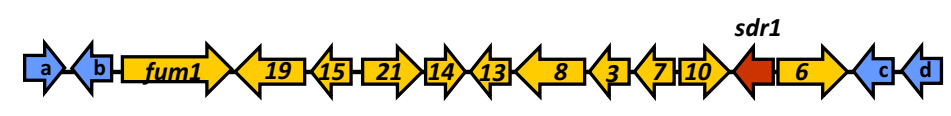

\section{A. niger ITEM 10355 FB nonproducer}
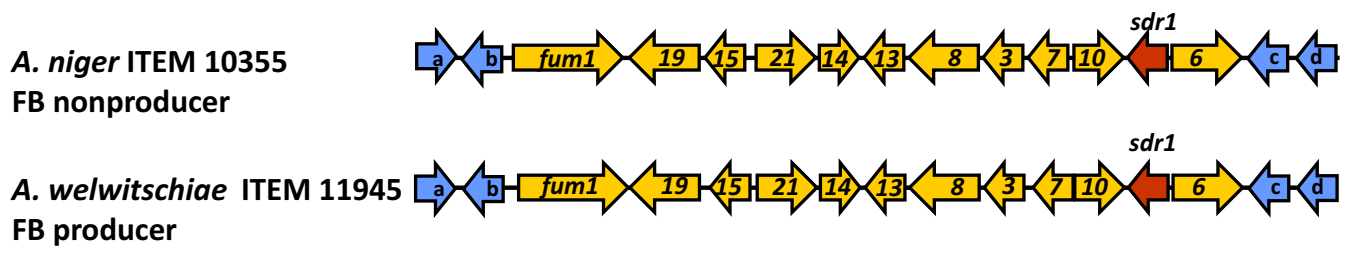
FB produ

\author{
A. welwitschiae ITEM 7468 \\ FB nonproducer
}

A. Iuchuensis CBS 106.47

A. Iuchuensis IFO 4308
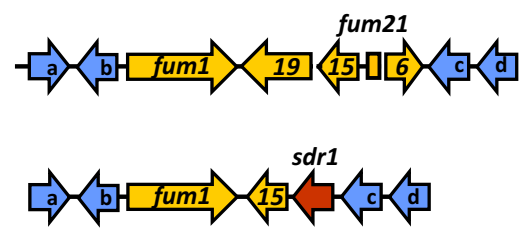

A. brasiliensis CBS 101740

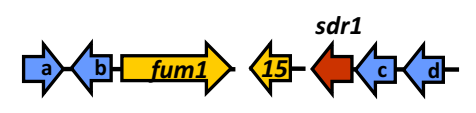

A. tubingensis CBS 134.48

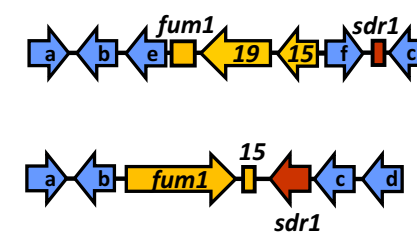

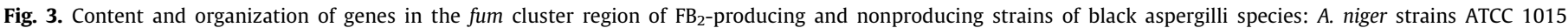

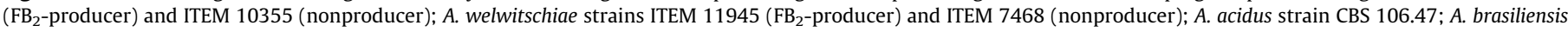

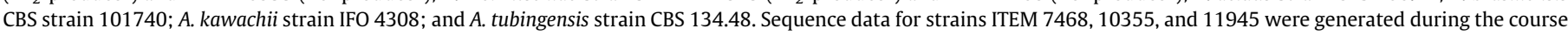

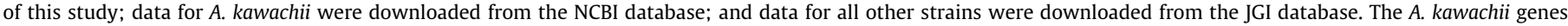

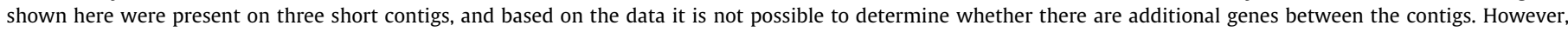

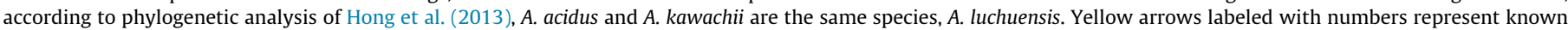

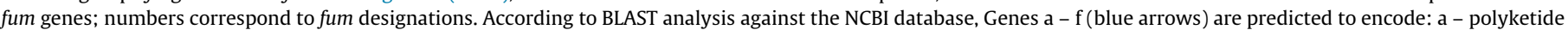

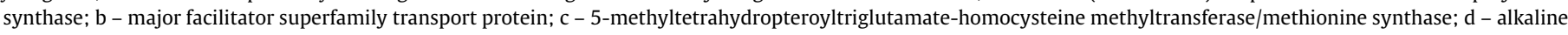

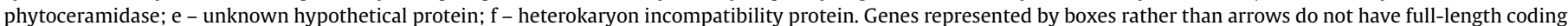

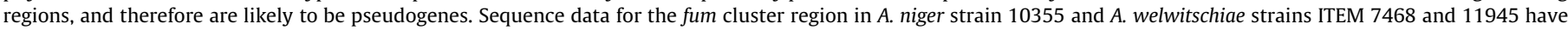
been submitted to NCBI/GenBank as accessions KJ934797, KJ934796 and KJ934798 respectively.
\end{abstract}

Table S1). These remnants are similar to the partially deleted cluster in A. welwitschiae in that they have retained all or part of fum1 and fum15, and in the case of A. brasiliensis all of fum19 (Fig. 3). In addition, genes immediately flanking the intact and partial clusters are conserved (Fig. 3), indicating that the genomic context, and therefore most likely the chromosomal location, of the intact and partial clusters is the same. The partial clusters in A. brasiliensis, A. luchuensis, and A. tubingensis differed from the partial cluster in A. welwitschiae in that they included all or part of $s d r 1$.

\subsection{Phylogenetic analysis of fum cluster sequences}

A subset of $13 \mathrm{~A}$. welwitschiae and $11 \mathrm{~A}$. niger isolates were selected to assess the phylogenetic relationships of fum genes in $\mathrm{FB}_{2}$-producing and nonproducing isolates. For this analysis, we employed DNA sequence data generated from fragments amplified in the PCR screen (Table 2) or from genome sequence data when available. The sequence analysis confirmed that the PCR amplicons were the target fum gene fragments. Because of the large deletion in the fum cluster and our PCR strategy, we had limited sequence data (only fum 1 and fum 15 fragments) for phylogenetic analyses that included $\mathrm{FB}_{2}$-nonproducing isolates of $A$. welwitschiae. Analyses of fum 1 and fum 15 alone or combined were consistent; internodes with significant support (i.e. bootstrap values $>70$ ) did not conflict between trees generated with the fum 1 and/or fum 15 datasets (Fig. 4, Supplemental Fig. S3). These datasets did not resolve either A. niger or A. welwitschiae isolates into a well-supported monophyletic clade; nor did they resolve $\mathrm{FB}_{2}$-producing and nonproducing isolates of $A$. niger into distinct clades (Fig. 4). However, the combined fum1-fum 15 dataset resolved $\mathrm{FB}_{2}$-producing isolates of $A$. welwitschiae into a well-supported monophyletic clade that did not include nonproducing isolates. $\mathrm{FB}_{2}$-nonproducing isolates of A. welwitschiae were not resolved into a distinct clade. In addition, in an unrooted tree generated from the combined fum1-fum15 dataset, the three isolates (ITEM 11448, ITEM 11552 and ITEM 11553) for which the species identity could not be determined were resolved as a well-supported clade (bootstrap value 99\%).

When $\mathrm{FB}_{2}$-nonproducing isolates of $A$. welwitschiae were excluded, we were able to conduct phylogenetic analyses with fragments of nine fum genes: fum1, fum15, fum21, fum14, fum13, fum8, fum3, fum7 and fum10. Well-supported internodes of individual unrooted trees were largely consistent with trees based on concatenated sequences of the nine genes (Fig. 5, Supplemental Fig. S3), but there were several exceptions to this. For example, the five $A$. niger isolates ITEM 5218, ITEM 5219, ITEM 7090, ITEM 7091 and ITEM 10355 were resolved into a single well-supported clade in some trees, but into different well-supported clades in others (Supplemental Fig. S3). In the analysis using concatenated fum sequences, all fumonisin-producing $A$. welwitschiae isolates were resolved into a well-supported monophyletic clade (Fig. 5). In trees generated with concatenated sequences [fum1, fum15, 


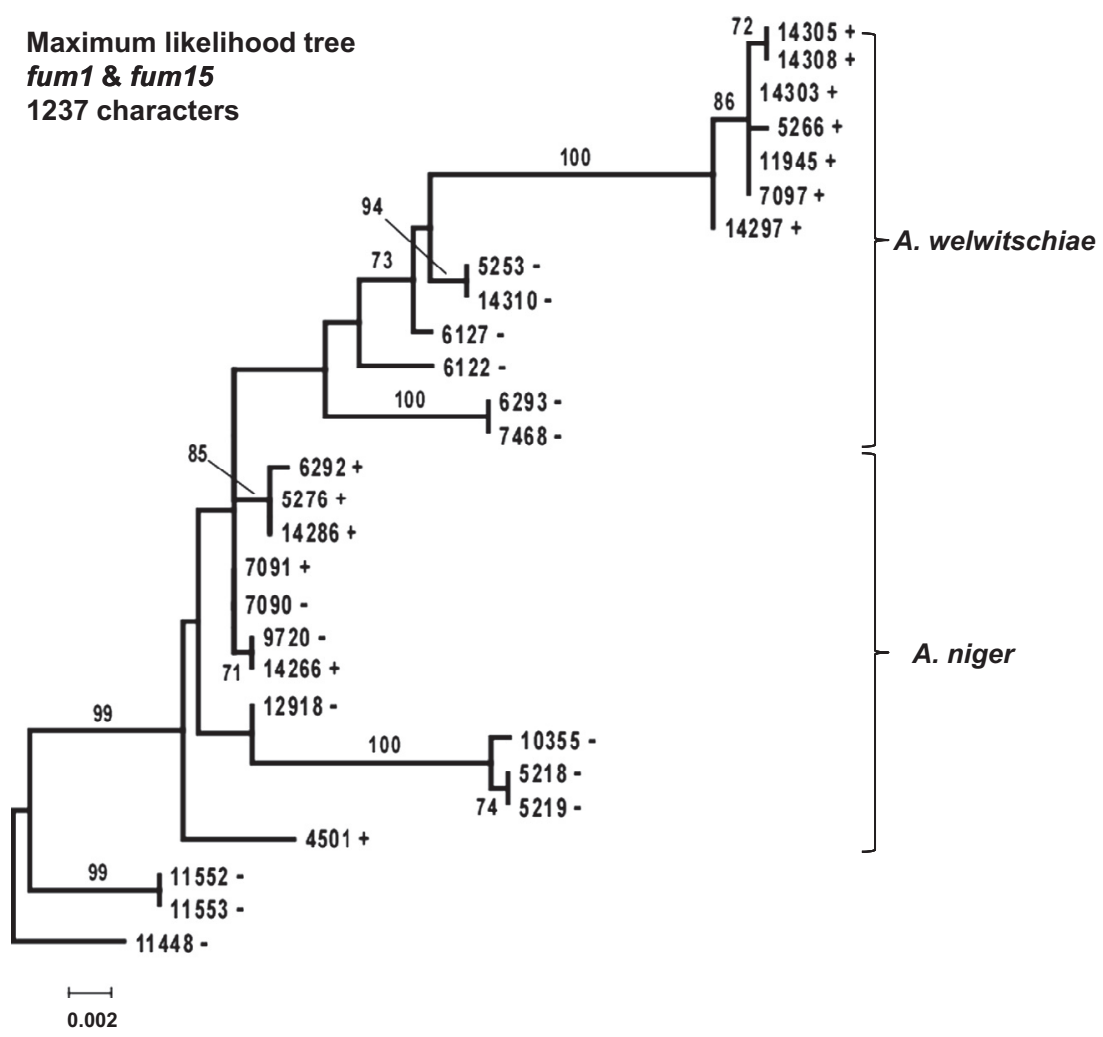

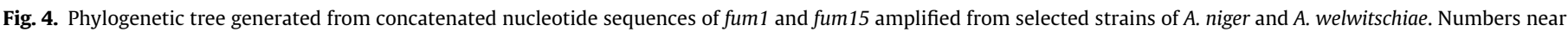

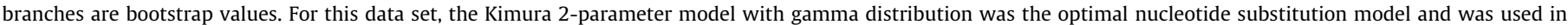

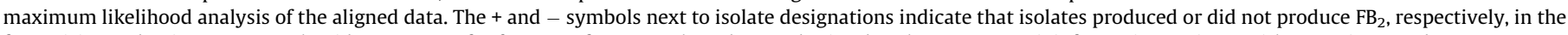

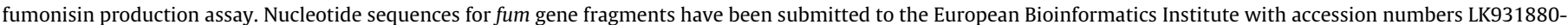
LK931897 (fum1) and LK931998-LK932015 (fum15).

fum21 (segments I and II), fum14, fum13, fum8, fum3, fum7 and fum 10] and rooted with $A$. welwitschiae sequences, all $A$. niger isolates were also resolved as a well-supported clade (Supplemental Fig. S4). In both unrooted (Fig. 5) and rooted (Supplemental Fig. S4) trees generated with the concatenated data set, A. niger isolates were resolved into two well-supported clades, both of which included at least one $\mathrm{FB}_{2}$-producing and multiple nonproducing isolates. In fact, some isolates of $A$. niger (e.g. isolates ITEM 7091 and ITEM 7090) with different fumonisin production phenotypes were more closely related to one another than to isolates with the same phenotype (Fig. 5). Thus, in A. niger there is evidence that fum sequences from $\mathrm{FB}_{2}$-producing and nonproducing isolates do not constitute two distinct clades.

We also conducted a phylogenetic analysis of full-length fum 1 sequences from black Aspergillus species from which sequence data for an intact or partial fum cluster was available. The truncated fum 1 sequence in $A$. brasiliensis was excluded from this analysis, because it corresponded to only $5 \%$ of the full-length fum 1 sequence. The results of this phylogenetic analysis indicate that fum 1 sequences from $A$. niger and $A$. welwitschiae are more closely related to one another than either is to sequences from A. luchuensis or A. tubingensis (Fig. 6). These results are consistent with results of phylogenetic analysis of combined sequences of benA, caM, and the rDNA internal spacer sequences (ITS) of black aspergilli that indicated $A$. niger and $A$. welwitschiae are more closely related to one another than either is to the three other species (Hong et al. 2013). The four fum 1 homologues from partial fum clusters used for the analysis shown in Fig. 6 appear to be functional, because their nucleotide sequences do not include frame shifts or internal stop codons. The exception is a single-nucleotide deletion near the $3^{\prime}$ end of the fum 1 homologue in A. welwitschiae strain ITEM 7468. This deletion is predicted to both truncate the corresponding protein by three amino acids and change the resulting five carboxy-terminal amino acids such that they are completely different from the amino acids at the equivalent position in other homologues.

When selection for a functional protein-encoding gene is maintained, nonsynonymous substitutions (i.e. mutations that alter amino acid sequence) are retained less frequently than synonymous substitutions (i.e. mutations that do not alter amino acid sequence) (Nei and Kumar, 2000). To assess whether selection for a functional fum 1 was relaxed or change following partial deletion of the cluster, we used the Selecton program (Stern et al., 2007) to estimate differences in the rates of synonymous and nonsynonymous substitutions along the entire length of the fum 1 coding region of the eight aspergilli isolates/species shown in Fig. 6. The results of the analysis provide evidence for purifying selection (i.e. selection against nucleotide changes that alter the amino acid sequence) at positions along the length of the fum1-encoded polyketide synthase and, therefore, selection against changes with the potential to negatively affect or change enzyme function.

\section{Discussion}

Production of fumonisin mycotoxins by black aspergilli is of concern because collectively these fungi can occur on a diversity of food and feed crops, can cause food spoilage, and are important for industrial fermentation, including production of compounds (e.g. citric acid) that are used in food. The results of the current study provide evidence that the predominant black aspergilli species on grapes in the Mediterranean Basin are A. niger and $A$. welwitschiae, two species that also predominate on grapes in California (Palumbo et al., 2013). The previous analysis of black 


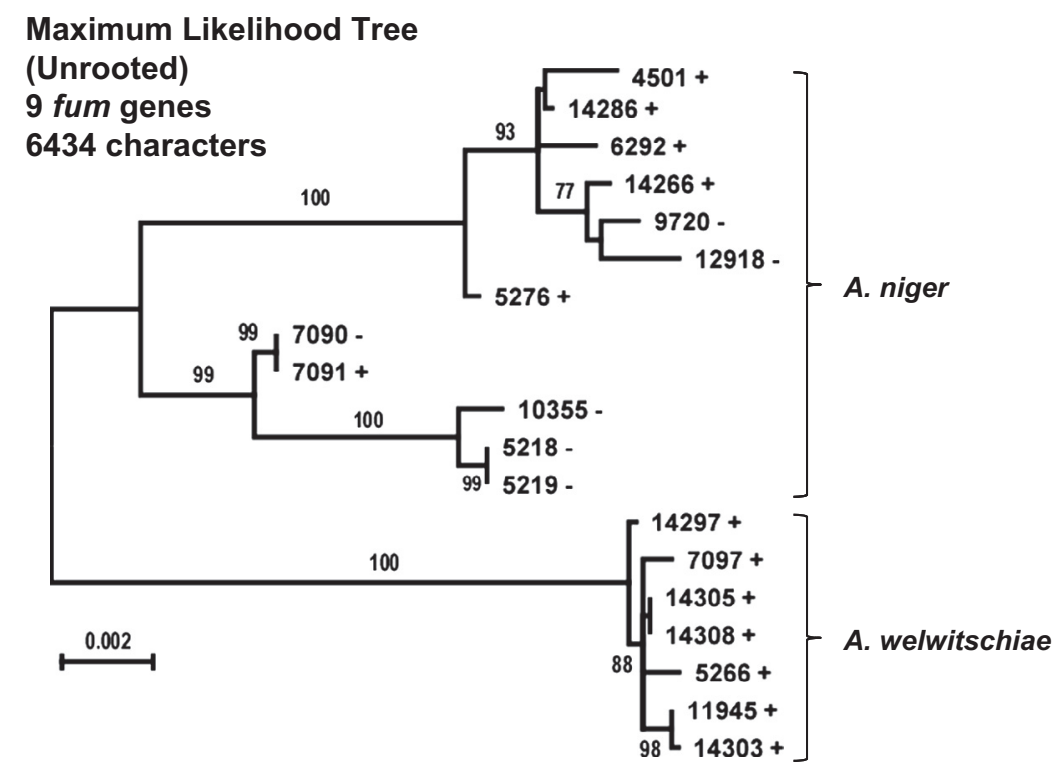

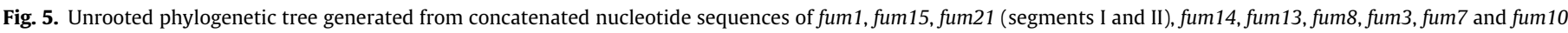

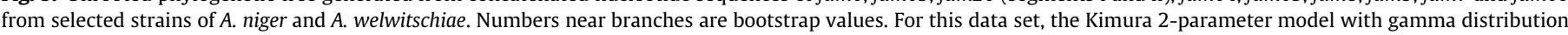

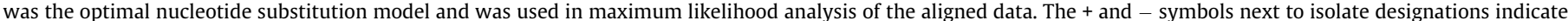

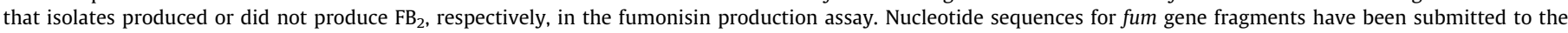

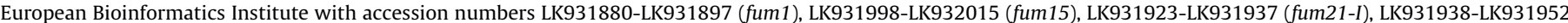

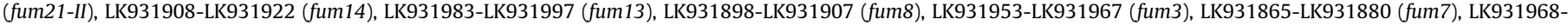
LK931982 (fum10).

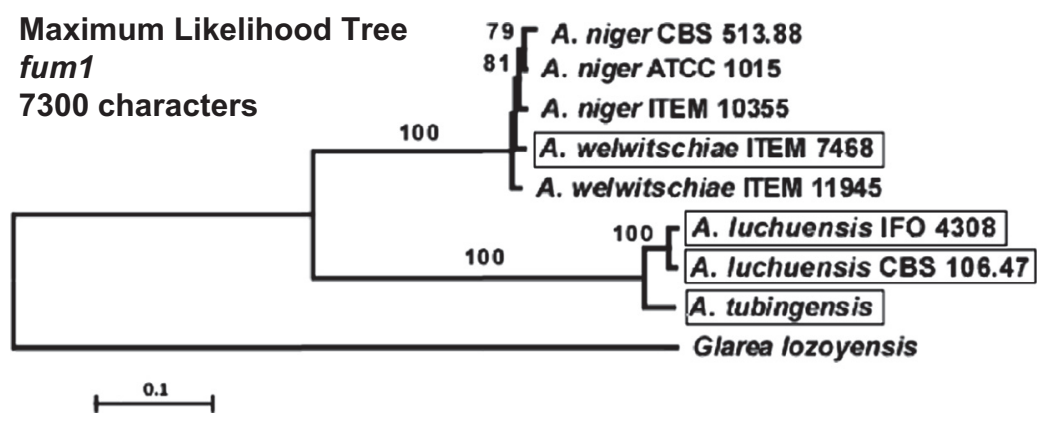

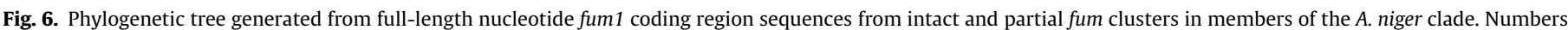

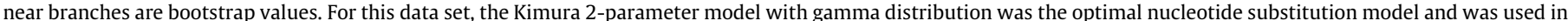
the maximum likelihood analysis of the aligned data. Boxes around names indicate species/strains with a partial fum cluster.

aspergilli from California grapes indicated that fumonisin-nonproduction was associated with the absence of six fum genes in A. welwitschiae but not in A. niger (Palumbo et al., 2013). Results of the current study further clarify the structure of the fum cluster in fumonisin-producing and nonproducing isolates of these species. The results of our analyses indicate that gene content and organization within the fum cluster is the same in fumonisin-producing isolates of both species and in nonproducing isolates of $A$. niger, but that the cluster has undergone an eight-gene deletion in nonproducing isolates of $A$. welwitschiae (Fig. 3, Tables 1 and 2).

The lack of $\mathrm{FB}_{2}$ nonproduction in some $A$. niger isolates that was observed in the current study is consistent with previous reports of fumonisin nonproduction in other isolates of this species (Frisvad et al., 2007; Palumbo et al., 2013; Varga et al., 2010). Because we examined production under only one culture condition (i.e. on CY20S medium), and because fumonisin production can vary under different conditions (Frisvad et al., 2007, 2011), our results do not exclude the possibility that $A$. niger isolates that did not produce $\mathrm{FB}_{2}$ on CY20S medium could produce $\mathrm{FB}_{2}$ on other media. Nevertheless, production of $0.5-3.3 \mu \mathrm{g} \mathrm{FB}$ per g CY20S culture in some isolates of $A$. niger that we examined and the lack of production in other isolates indicate a fundamental difference(s) between these two groups of isolates. Comparisons of fum cluster sequences in the $\mathrm{FB}_{2}$-producing and nonproducing isolates of $A$. niger do not provide definitive evidence for the genetic basis for the difference and raise the possibility that the cause of nonproduction may differ among isolates. The data suggest that nonproduction could result from four types of genetic modifications: (i) a small deletion within a fum gene coding region that renders the corresponding protein nonfunctional; (ii) a base substitution in a fum gene coding region that introduces a premature stop codon or causes an amino acid substitution that renders the corresponding protein nonfunctional; (iii) a base substitution in a fum cluster regulatory sequence that disrupts fum gene expression; or (iv) a mutation outside the fum cluster that negatively affects fumonisin production, e.g. by blocking expression of cluster genes. Evidence for nonproduction resulting from a small deletion within a fum gene is the 12-base deletion in the fum 1 homologue of $A$. niger isolate ITEM 10355. This deletion is located between the predicted $\beta$-ketoacyl synthase and acyl transferase domains of the fum1-encoded polyketide synthase (Proctor et al., 1999), and although these two domains are essential for function of the polyketide synthase, it is unclear whether a 
small in-frame deletion between them could affect function. In addition, the fum 1 deletion occurs in a region that includes multiple in-frame deletions/insertions of $>20$ bases in fum 1 homologues in other fungi, e.g. Aspergillus, Fusarium, Glarea, and Tolypocladium (data not shown). Thus the deletion in ITEM 10355 would not necessarily affect the functionality of the polyketide synthase, and therefore, the ability of ITEM 10355 to produce fumonisins. The only evidence that a base substitution within a fum gene coding region could contribute to $\mathrm{FB}_{2}$ nonproduction is the substitution and resulting premature stop codon in fum10 of isolate ITEM 12918. In Fusarium, the fum 10 homologue is required for formation of the tricarboxyl esters of fumonisins and, therefore, for production of $\mathrm{FB}_{2}$ or $\mathrm{FB}_{4}$. However, fum10 is not required for synthesis of fumonisin biosynthetic intermediates known as hydrolyzed fumonisins, because they lack the tricarboxyl esters (Butchko et al., 2006). Assuming fum10 has the same function in Aspergillus and Fusarium, it is possible that isolate ITEM 12918 produces hydrolyzed fumonisins. Examination of ITEM 12918 for production of hydrolyzed fumonisins could help resolve the role of the premature stop codon in fum 10 in the $\mathrm{FB}_{2}$-nonproduction phenotype in this strain.

Data from the current study do not directly address the possibility that fumonisin nonproduction in $A$. niger results from a mutation of a regulatory element within or outside the fum cluster. However, a previous study of $A$. niger revealed that the level of expression of eight cluster genes was relatively high in a fumonisin-producing isolate, whereas expression of some fum genes was markedly reduced in nonproducing isolates (Palumbo et al., 2013). In some isolates, expression of genes (e.g. fum1 and fum8) that are essential for formation of the fumonisin backbone was not detected. Although environmental conditions can have differing effects on fumonisin production in Aspergillus and Fusarium (Mogensen et al., 2009), common themes in the genetic mechanisms that regulate fungal secondary metabolism (Keller et al., 2005 ) indicate that regulation of fum gene expression in Aspergillus and Fusarium likely includes some of the same genetic and physiological components. In Fusarium, the FUM cluster gene FUM21 encodes a transcription factor that induces expression of other cluster genes (Brown et al., 2007). Examination of the full-length fum21 sequence in $A$. niger nonproducing isolate ITEM 10355 did not reveal any deletions, insertions or base substitutions that would have an obvious negative affect on its activity. Fusarium fum gene homologues are also regulated by proteins encoded by genes located outside the cluster. These proteins include transcription factors that regulate carbon and nitrogen metabolism and proteins involved in methylation and acetylation of chromatin and thereby modification of the accessibility of DNA to transcriptional machinery (Woloshuk and Shim, 2013). Homologues of such regulatory genes are present in Aspergillus and could contribute to regulation of fumonisin production in A. niger. Based on current data, however, it is not possible to draw any conclusions as to whether such regulators contribute to the fumonisin-nonproduction phenotype in isolates of $A$. niger.

The close phylogenetic relationships of fum genes in some fumonisin-producing and nonproducing isolates of $A$. niger (Fig. 5) is consistent with nonproduction resulting from a mutation in a regulatory gene located outside the cluster or with the hypothesis that nonproduction has arisen multiple times in $A$. niger by small mutations within the fum cluster. The 12-base deletion in fum 1 of ITEM 10355 and the premature stop codon in fum 10 of ITEM 12918 indicate that different nonproducing isolates of $A$. niger can have different mutations that potentially affect fumonisin production. Thus, the genetic basis for fumonisin nonproduction in A. niger remains unclear. Functional analyses could help to resolve this issue. For example, if nonproduction is the result of the 12-base deletion in the fum 1 homologue of ITEM 10355 , transformation-mediated introduction of a fum 1 homologue from a producing isolate into this isolate should restore fumonisin production. However, if nonproduction is the result of a mutation outside the cluster, it should be possible to restore production by inducing expression of fum cluster genes, e.g. by constitutive expression of the cluster regulatory gene, fum 21 .

The eight-gene deletion in fumonisin-nonproducing isolates of $A$. welwitschiae is consistent with the hypothesis that the absence of fum genes is the genetic basis for nonproduction in this species (Palumbo et al., 2013), because Fusarium orthologues of some of the deleted genes are required for synthesis of $\mathrm{FB}_{2}$ (Alexander et al., 2009; Uhlig et al., 2012; Du et al., 2008). While partial deletion of the cluster would prevent fumonisin biosynthesis from occurring and provides a straightforward explanation for nonproduction in $A$. welwitschiae, the presence of the deletion does not rule out the possibility that the original genetic cause of nonproduction was a mutation(s) in a regulatory gene located outside the fum cluster, and that deletion of fum genes occurred after mutation of the regulatory gene. As with $A$. niger, functional analyses could provide further insight into the genetic basis of fumonisin nonproduction in $A$. welwitschiae. For example, if nonproduction is due solely to the partial deletion, transformation-mediated introduction of a functional fum cluster from a producing isolate into a nonproducing isolate should restore fumonisin production.

The phylogenetic analysis of benA, caM and ef- $1 \alpha$ resolved a clade within A. welwitschiae that consisted of both fumonisin-producing and nonproducing isolates (Fig. 1). This indicates that isolates with different production abilities can be more closely related to one another than isolates with the same production ability, and therefore, that this species is not divided into two diverging lineages, one consisting of only producing isolates and the other of only nonproducing isolates. In contrast to the housekeeping genes, phylogenetic analysis of fum 1 and fum 15 resolved all of the fumonisin-producing isolates of $A$. welwitschiae into a wellsupported clade that did not include nonproducing isolates (Fig. 4). Together, the resolution of producing isolates into a single clade in the fum $1 /$ fum 15 phylogeny, the identical pattern of fum gene loss in nonproducing isolates, and the identical genomic context of the fum cluster in producing and nonproducing isolates suggest that the intact and partial clusters constitute alternate alleles of a fum cluster locus. However, although the partial fum cluster homologues in A. welwitschiae isolates appear to constitute the same allele based on gene content and organization, the fum1fum15 sequences from partial clusters were not resolved into a well-supported monophyletic clade in the gene tree (Fig. 4). Thus, there may be multiple partial fum clusters alleles in A. welwitschiae.

The phylogenetic trees inferred from housekeeping genes (Fig. 1) suggest that relatively closely related isolates of $A$. welwitschiae can have different fum cluster alleles and that relatively distantly related isolates can have the same allele. The presence of different alleles of a locus in closely related individuals of a species is indicative of genetic recombination. A likely mechanism for such recombination is heterothallic sexual reproduction, i.e. when two distantly related individuals with different alleles undergo sexual reproduction they can give rise to closely related individuals (i.e. progeny) with the different alleles. Although, a sexual cycle has not been reported in $A$. welwitschiae, one has been reported in the black Aspergillus species A. tubingensis (Horn et al., 2013) as well as several other aspergilli that were once considered to be asexual (Horn et al., 2009). In addition, formation of sclerotia, a prerequisite for sexual reproduction in multiple aspergilli, have been induced in $A$. niger and some other black aspergilli, although not yet in A. welwitschiae (Frisvad et al., 2014). Furthermore, a full complement of early sexual development genes, including the mat1 mating-type gene, are present in genome sequences of $A$. niger (Pel et al., 2007). Thus, it is possible that an as yet unreported 
sexual cycle has contributed the presence of the different fum cluster alleles in closely related isolates of $A$. welwitschiae. If this is the case, it would be less likely that the original genetic cause of nonproduction in A. welwitschiae is a mutation in a regulatory gene located outside the cluster, because genetic recombination during meiosis could give rise to fumonisin-nonproducing progeny with the mutated regulatory gene and an intact fum cluster. Such a scenario is not consistent with the finding that all fumonisin nonproducing isolates of $A$. welwitschiae examined to date lack some fum genes (Palumbo et al., 2013; and Table 2 of current study).

The results of the current and previous studies indicate that on grape $A$. niger and $A$. welwitschiae consist of mixed populations of fumonisin-producing and nonproducing individuals. Based on current data, we propose the following hypothesis for how the mixed population arose in A. welwitschiae: after A. welwitschiae diverged from $A$. niger, the fum cluster underwent an eight-gene deletion in one or more strains; subsequently, both partially deleted and intact forms of the cluster were retained and exchanged through genetic recombination, possibly by meiosis. More extensive analyses of housekeeping genes and the fum cluster from more isolates of $A$. welwitschiae should provide additional support for or against this hypothesis. Although existing data do not point to a clear hypothesis for how the mixed population of fumonisin-producing and nonproducing isolates arose in $A$. niger, the data indicate two fundamental differences from how such a mixture arose in $A$. welwitschiae. First, the mixed population in A. niger was not facilitated by a large deletion within the fum cluster. Second, the close phylogenetic relationships of fum sequences in some producing and nonproducing isolates of $A$. niger (Fig. 5) suggest recent exchange of fum sequences between fumonisin-producing and nonproducing individuals and possibly that the fum gene mutation(s) responsible for nonproduction occurred relatively recently. In A. welwitschiae, by contrast, fum sequences from producing strains are relatively distantly related to those of nonproducing strains. There is evidence for variation in secondary metabolite production phenotypes (chemotypes) among individuals in other fungal species. For example, the ability to produce aflatoxins varies among isolates of A. flavus, and likely results from deletion of genes or point mutations within the aflatoxin biosynthetic gene (afl) cluster (Chang et al., 2005; Mauro et al., 2013; Moore et al., 2009). In addition, isolates of some Fusarium species can differ in the kinds of trichothecene mycotoxins (e.g. nivalenol, 3-acetyl deoxynivalenol, or 15 -acetyl deoxynivalenol) they produce as a result of loss or variation in function of trichothecene biosynthetic genes (Kimura et al., 2003; Lee et al., 2002; Ward et al., 2002). Thus, the variation in fumonisin production ability in A. niger and A. welwitschiae provides a further example of intra-species variation in mycotoxin chemotypes among fungi.

The $A$. niger clade of black aspergilli consists of at least 12 phylogenetically distinct species, including $A$. brasiliensis, A. luchuensis, A. niger, A. tubingensis, and A. welwitschiae (Hong et al., 2013). The similarities in gene content of the partial fum clusters in these species (Fig. 3) suggests that the partial cluster could have resulted from a deletion event in their common ancestor followed by vertical inheritance of the resulting partial cluster through multiple speciation events. In this scenario, partial deletion would have occurred prior to speciation, and as a result, fum sequences from intact versus partial clusters in the same species should be more distantly related to one another than fum sequences from partial clusters from different species. But, this is not the case; the fum 1 homologue from the partial $A$. welwitschiae cluster is more closely related to the homologue from the intact $A$. welwitschiae cluster than to homologues from partial clusters in other species (Fig. 6). This suggests that the deletion(s) resulting in the partial fum cluster in A. welwitschiae occurred after this species diverged from other species with partial clusters. An alternative explanation for the similarity in gene content of partial fum cluster orthologues is non-random loss of genes in different species. There is evidence for non-random gene loss in the afl cluster among isolates of A. flavus (Chang et al., 2005; Mauro et al., 2013; Moore et al., 2009) and in the gibberellic acid (GA) biosynthetic gene cluster within and among species of Fusarium (Bömke and Tudzynski, 2009; Wiemann et al., 2013). For both the afl and GA clusters, some of the deleted genes are responsible for early steps in the biosynthetic pathways such that the pathways are blocked before metabolites with aflatoxin- or GA-like structures are formed. This suggests that the non-random loss could reflect selection for isolates that do not produce aflatoxin- and GA-like metabolites. The apparent non-random gene loss within the Aspergillus fum cluster differs in that a full-length fum 1 has been retained in the partial clusters of the species examined except for $A$. brasiliensis. The fum1-encoded polyketide synthase is responsible for the first step in fumonisin biosynthesis, namely synthesis of the linear polyketide that forms the backbone of the fumonisin structure (Alexander et al., 2009; Du et al., 2008). Even though other genes (e.g. fum6, fum8, and fum21) that are absent in the partial clusters are also necessary for formation of fumonisin-like metabolites (Alexander et al., 2009; Uhlig et al., 2012; Du et al., 2008), if non-random gene loss were due only to selection for loss of production of fumonisin-like molecules, loss of the fum 1 side of the cluster would be expected to occur in some species. Thus, non-random gene loss in fum cluster orthologs among black aspergilli could result from selection for a functional fum 1 and production of the corresponding polyketide. This hypothesis is consistent with the absence of frame shift mutations and of nucleotide changes that introduce premature stop codons in the coding region of fum 1 homologues from all partial clusters except for the A. brasiliensis fum 1 homologue. In addition, the hypothesis is consistent with results indicating selection against nucleotide changes that alter the amino acid sequence, and potentially function, of the fum1-encoded enzyme (i.e. purifying selection) regardless of whether fum1 was in an intact or partial cluster (Supplemental Table S2). However, the latter results (Supplemental Fig. S5) should be interpreted with caution, because if partial deletion of the cluster and relaxation of selection had occurred relatively recently, most of the evolutionary history of fum 1 would have occurred in the presence of selection, and as a result the signature of purifying selection in fum1 would likely still persist. Given that A. brasiliensis, A. luchuensis, A. niger, A. tubingensis, and A. welwitschiae represent a large proportion of the phylogenetic diversity of the A. niger clade (Hong et al., 2013), it is possible that other species in this clade also have an intact or partial fum cluster. Furthermore, given the evidence that in at least some cases partial deletion of the cluster occurred after speciation, it is also possible that the cluster and the ability to produce fumonisins were once more widespread in this group of fungi than they currently are.

The results of the current study provide further insight into the evolutionary history and the genetic bases for variation in secondary metabolite production within fungal species. This insight includes evidence suggesting that a sexual cycle has contributed to variation in fumonisin production in the black Aspergillus species A. welwitschiae, evidence that is consistent with reports of sexual reproduction in other Aspergillus species once considered to be asexual. Analysis of mating type genes, sexual reproduction, and functional analyses of fum clusters should provide further insight into the genetic basis for variation in fumonisin production in black aspergilli and further contribute to diagnostic methods to distinguish between producing and nonproducing isolates.

\section{Acknowledgments}

The authors gratefully acknowledge Alessandra Villani, Vincenzo Ricci, Marcie Moore, and Crystal Probyn for their 
excellent technical support, to Todd J. Ward for helpful comments on phylogenetic analyses and the manuscript, and to projects FP7 MYCORED and CISIA for financial support.

\section{Appendix A. Supplementary material}

Supplementary data associated with this article can be found, in the online version, at http://dx.doi.org/10.1016/j.fgb.2014.09.009.

\section{References}

Abarca, M.L., Accensi, F., Bragulat, M.R., Castella, G., Cabanes, F.J., 2003. Aspergillus carbonarius as the main source of ochratoxin A contamination in dried wine fruits from the Spanish market. J. Food Prot. 66, 504-506.

Abarca, M.L., Accensi, F., Cano, J., Cabanes, F.J., 2004. Taxonomy and significance of black aspergilli. Antonie Van Leeuwenhoek 86, 33-49.

Abrunhosa, L., Calado, T., Venancio, A., 2011. Incidence of fumonisin B(2) production by Aspergillus niger in Portuguese wine regions. J. Agric. Food Chem. 59, 7514 7518.

Alexander, N.J., Proctor, R.H., Mc Cormick, S.P., 2009. Genes, gene clusters, and biosynthesis of trichothecenes and fumonisins in Fusarium. Toxin Rev. 28, 198215.

Altschul, S.F., Gish, W., Miller, W., Myers, E.W., Lipman, D.J., 1990. Basic local alignment search tool. J. Mol. Biol. 215, 403-410.

Andersen, M.R., Salazar, M.P., Schaap, P.J., van de Vondervoort, P.J., Culley, D., Thykaer, J., Frisvad, J.C., Nielsen, K.F., Albang, R., Albermann, K., Berka, R.M., Braus, G.H., Braus-Stromeyer, S.A., Corrochano, L.M., Dai, Z., van Dijck, P.W., Hofmann, G., Lasure, L.L., Magnuson, J.K., Menke, H., Meijer, M., Meijer, S.L. Nielsen, J.B., Nielsen, M.L., van Ooyen, A.J., Pel, H.J., Poulsen, L., Samson, R.A., Stam, H., Tsang, A., van den Brink, J.M., Atkins, A., Aerts, A., Shapiro, H., Pangilinan, J., Salamov, A., Lou, Y., Lindquist, E., Lucas, S., Grimwood, J. Grigoriev, I.V., Kubicek, C.P., Martinez, D., van Peij, N.N., Roubos, J.A., Nielsen, J., Baker, S.E., 2011. Comparative genomics of citric-acid-producing Aspergillus niger ATCC 1015 versus enzyme-producing CBS 513.88. Genome Res. 21, 885897.

Baker, S.E., 2006. Aspergillus niger genomics: past, present and into the future. Med. Mycol. 44, S17-S21.

Bömke, C., Tudzynski, B., 2009. Diversity, regulation, and evolution of the gibberellin biosynthetic pathway in fungi compared to plants and bacteria. Phytochemistry 70, 1876-1893.

Brown, D.W., Butchko, R.A.E., Busman, M., Proctor, R.H., 2007. The Fusarium verticillioides FUM gene cluster encodes a Zn(II)2Cys6 protein that affects FUM gene expression and fumonisin production. Eukaryot. Cell 6, 1210-1218.

Butchko, R.A.E., Plattner, R.D., Proctor, R.H., 2006. Deletion analysis of FUM genes involved in tricarballylic ester formation during fumonisin biosynthesis. J. Agric Food Chem. 54, 9398-9404.

Cabanes, F.J., Accensi, F., Bragulat, M.R., Abarca, M.L., Castella, G., Minguez, S., Pons, A., 2002. What is the source of ochratoxin A in wine? Int. J. Food Microbiol. 79, $213-215$.

Chang, P.K., Horn, B.W. Dorner, J.W., 2005. Sequence breakpoints in the aflatoxin biosynthesis gene cluster and flanking regions in nonaflatoxigenic Aspergillus flavus isolates. Fun. Genet. Biol. 42, 914-923.

De Girolamo, A., Pascale, M., Visconti, A., Solfrizzo, M., 2011. Comparison of methods and optimisation of the analysis of fumonisins B1 and B2 in masa flour, and alkaline cooked corn product. Food Addit. Contam. Part A: Chem. Anal. Control Expo. Risk Assess. 28, 667-675.

Du, L., Gerber, R., Huffman, J., Lou, L., Jorgenson, J., Yu, F., Zaleta-Rivera, K., Wang, O. 2008. Biosynthesis of sphinganine-analog mycotoxins. J. Ind. Microbiol. Biotechnol. 35, 455-464.

Felsenstein, J., 1985. Confidence limits on phylogenies: an approach using the bootstrap. Evolution 39, 783-791.

Frisvad, J.C., Smedsgaard, J., Samson, R.A., Larsen, T.O., Thrane, U., 2007. Fumonisin B2 production by Aspergillus niger. J. Agric. Food Chem. 55, 9727-9732.

Frisvad, J.C., Larsen, T.O., Thrane, U., Meijer, M., Varga, J., Samson, R.A., Nielsen, K.F. 2011. Fumonisin and ochratoxin production in industrial Aspergillus niger strains. PLoS One 6, e23496.

Frisvad, J.C., Petersen, L.M., Lyhne, E.K., Larsen, T.O., 2014. Formation of sclerotia and production of indoloterpenes by Aspergillus niger and Other Species in Section Nigri. PLoS One 9, e94857.

Futagami, T., Mori, K., Yamashita, A., Wada, S., Kajiwara, Y., Takashita, H., Omori, T. Takegawa, K., Tashiro, K., Kuhara, S., Goto, M., 2011. Genome sequence of the white koji mold Aspergillus kawachii IFO 4308, used for brewing the Japanese distilled spirit shochu. Eukaryot. Cell 10, 1586-1587.

Gams, W., Christensen, M., Onions, A.H., Pitt, J.I., Samson, R.A., 1985. Infrageneric taxa of Aspergillus. In: Samson, R.A., Pitt, J.I. (Eds.), Advances in Penicillium and Aspergillus Systematics. Plenum Press, New York, pp. 55-62.

Glass, N.L., Donaldson, G.C., 1995. Development of primer sets designed for use with the PCR to amplify conserved genes from filamentous ascomycetes. Appl. Environ. Microbiol. 61, 1323-1330.

Hong, S.B., Lee, M., Kim, D.H., Varga, J., Frisvad, J.C., Perrone, G., Gomi, K., Yamada, O., Machida, M., Houbraken, J., Samson, R.A., 2013. Aspergillus luchuensis, an industrially important black Aspergillus in East Asia. PLoS One 8, e63769.
Horn, B.W., Moore, G.G., Carbone, I., 2009. Sexual reproduction in Aspergillus flavus. Mycologia 101, 423-429.

Horn, B.W., Olarte, R.A., Peterson, S.W., Carbone, I., 2013. Sexual reproduction in Aspergillus tubingensis from section Nigri. Mycologia 105, 1153-1163.

Keller, N.P., Turner, G., Bennett, J.W., 2005. Fungal secondary metabolism - from biochemistry to genomics. Nat. Rev. Microbiol. 3, 937-947.

Kimura, M., Tokai, T., O’Donnell, K., Ward, T.J., Fujimura, M., Hamamoto, H., Shibata, T., Yamaguchi, I., 2003. The trichothecene biosynthesis gene cluster of Fusarium graminearum F15 contains a limited number of essential pathway genes and expressed non-essential genes. FEBS Lett. 539, 105-110.

Lee, T., Han, Y.K., Kim, K.H., Yun, S.H., Lee, Y.W., 2002. Tri13 and Tri7 determine deoxynivalenol- and nivalenol-producing chemotypes of Gibberella zeae. Appl. Environ. Microbiol. 68, 2148-2154.

Logrieco, A., Ferracane, R., Haidukowsky, M., Cozzi, G., Visconti, A., Ritieni, A., 2009. Fumonisin $\mathrm{B}(2)$ production by Aspergillus niger from grapes and natural occurrence in must. Food Addit. Contam. 26, 1495-1500.

Logrieco, A., Ferracane, R., Visconti, A., Ritieni, A., 2010. Natural occurrence of fumonisin $\mathrm{B}(2)$ in red wine from Italy. Food Addit. Contam. 8, 1136-1141.

Mansson, M., Klejnstrup, M.L., Phipps, R.K., Nielsen, K.F., Frisvad, J.C., Gotfredsen, C.H., Larsen, T.O., 2010. Isolation and NMR characterization of fumonisin B2 and a new fumonisin B6 from Aspergillus niger. J. Agric. Food Chem. 58, 949-953.

Marasas, W.F.O., Riley, R.T., Hendricks, K., Stevens, V.L., Sadler, T.W., Gelineau-van Waes, J., Missmer, S.A., Cabrera, J., Torres, O., Gelderblom, W.C.A., Allegood, J. Martínez, C., Maddox, J., Miller, J.D., Starr, L., Sullards, M.C., Roman, A.V., Voss, K.A., Wang, E., Merrill, A.H., 2004. Fumonisins disrupt shingolipid metabolism, folate transport and neural tube development in embryo culture and in vivo: a potential risk factor for human neural tube defects among populations consuming fumonisin-contaminated maize. J. Nutri. 134, 711-716.

Mauro, A., Battilani, P., Callicott, K.A., Giorni, P., Pietri, A., Cotty, P.J., 2013. Structure of an Aspergillus flavus population from maize kernels in northern Italy. Int. J. Food Microbiol. 162, 1-7.

Mogensen, J.M., Nielsen, K.F., Samson, R.A., Frisvad, J.C., Thrane, U., 2009. Effect of temperature and water activity on the production of fumonisins by Aspergillus niger and different Fusarium species. BMC Microbiol. 9, 281.

Mogensen, J.M., Frisvad, J.C., Thrane, U., Nielsen, K.F., 2010. Production of fumonisin B2 and B4 by Aspergillus niger on grapes and raisins. J. Agric. Food Chem. 58, 954-958.

Moore, G.G., Singh, R., Horn, B.W., Carbone, I., 2009. Recombination and lineagespecific gene loss in the aflatoxin gene cluster of Aspergillus flavus. Mol. Ecol. 18, 4870-4887.

Nei, M., Kumar, S., 2000. Molecular Evolution and Phylogenetics. Oxford University Press, New York.

Noonim, P., Mahakarnchanakul, W., Nielsen, K.F., Frisvad, J.C., Samson, R.A., 2009. Fumonisin B2 production by Aspergillus niger in Thai coffee beans. Food Addit. Contam. Part A: Chem. Anal. Control Expo. Risk Assess. 26, 94-100.

O’Donnell, K., Nirenberg, H.I., Aoki, T., Cigelnik, E., 2000. A multigene phylogeny of the Gibberella fujikuroi species complex: detection of additional phylogenetically distinct species. Mycoscience 41, 61-78.

Palumbo, J.D., O'Keeffe, T.L., McGarvey, J.A., 2011. Incidence of fumonisin B2 production within Aspergillus section Nigri populations isolated from California raisins. J. Food Prot. 74, 672-675.

Palumbo, J.D., O'Keeffe, T.L., Gorski, L., 2013. Multiplex PCR analysis of fumonisin biosynthetic genes in fumonisin-nonproducing Aspergillus niger and A. awamori strains. Mycologia 105, 277-284.

Pel, H.J., de Winde, J.H., Archer, D.B., Dyer, P.S., Hofmann, G., Schaap, P.J., Turner, G., de Vries, R.P., Albang, R., Albermann, K., Andersen, M.R., Bendtsen, J.D., Benen, J.A., van den Berg, M., Breestraat, S., Caddick, M.X. Contreras, R., Cornell, M., Coutinho, P.M., Danchin, E.G., Debets, A.J., Dekker, P., van Dijck, P.W., van Dijk, A., Dijkhuizen, L., Driessen, A.J., d'Enfert, C., Geysens, S., Goosen, C., Groot, G.S., de Groot, P.W., Guillemette, T., Henrissat, B., Herweijer, M., van den Hombergh, J.P., van den Hondel, C.A., van der Heijden, R.T., van der Kaaij, R.M., Klis, F.M., Kools, H.J., Kubicek, C.P., van Kuyk, P.A., Lauber, J., Lu, X., van der Maarel, M.J. Meulenberg, R., Menke, H., Mortimer, M.A., Nielsen, J., Oliver, S.G., Olsthoorn, M., Pal, K., van Peij, N.N., Ram, A.F., Rinas, U., Roubos, J.A., Sagt, C.M., Schmoll, M., Sun, J., Ussery, D., Varga, J., Vervecken, W., van de Vondervoort, P.J., Wedler, H., Wosten, H.A., Zeng, A.P., van Ooyen, A.J., Visser, J., Stam, H., 2007. Genome sequencing and analysis of the versatile cell factory Aspergillus niger CBS 513.88. Nat. Biotechnol. 25, 221-231.

Perrone, G., Stea, G., Epifani, F., Varga, J., Frisvad, J.C., Samson, R.A., 2011. Aspergillus niger contains the cryptic phylogenetic species A. awamori. Fungal Biol. 115, $1138-1150$.

Pitt, J.I., Hocking, A.D., 2007. Fungi and Food Spoilage, second ed. Blackie, London. Pitt, J.I., Hocking, A.D., 2009. Fungi and Food Spoilage, third ed. Springer, London. Proctor, R.H., Desjardins, A.E., Plattner, R.D., Hohn, T.M., 1999. A polyketide synthase gene required for biosynthesis of fumonisin mycotoxins in Gibberella fujikuroi mating population A. Fungal Genet. Biol. 27, 100-112.

Proctor, R.H., Plattner, R.D., Desjardins, A.E., Busman, M., Butchko, R.A.E., 2006. Fumonisin production in the maize pathogen Fusarium verticillioides: genetic basis of naturally occurring chemical variation. J. Agric. Food Chem. 54, 2424-2430.

Raper, K.B., Fennell, D.I., 1965. The Genus Aspergillus. Williams \& Wilkins Company, Baltimore.

Rheeder, J.P., Marasas, W.F.O., Thiel, P.G., Sydenham, E.W., Shephard, G.S., van Schalkwyk, D.J., 1992. Fusarium moniliforme and fumonisins in corn in relation to human esophageal cancer in Transkei. Phytopathology 82, 353-357.

Sage, L., Garon, D., Seigle Murandi, F., 2004. Fungal microflora and ochratoxin A risk in French wineyards. J. Agric. Food Chem. 52, 5764-5768. 
Sambrook, J., Fritsch, E.F., Maniatis, T., 1989. Molecular Cloning, A Laboratory Manual, second ed. Cold Spring Harbor Laboratory Press, Plainview, New York. Samson, R.A., Hoekstra, E.S., Frisvad, J.C., 2004. Introduction to Food-and Airborne Fungi, seventh ed. Centraalbureau voor Schimmelcultures, Utrecht.

Stern, A., Doron-Faigenboim, A., Erez, E., Martz, E., Bacharach, E., Pupko, T., 2007. Selecton 2007: advanced models for detecting positive and purifying selection using a Bayesian inference approach. Nucleic Acids Res. 35, W506-W511.

Susca, A., Proctor, R.H., Mulè, G., Stea, G., Ritieni, A., Logrieco, A., Moretti, A., 2010 Correlation of mycotoxin fumonisin B2 production and presence of the fumonisin biosynthetic gene fum8 in Aspergillus niger from grape. J. Agric. Food Chem. 58, 9266-9272.

Tamura, K. Peterson, D., Peterson, N., Stecher G., Nei, M., Kumar, S., 2011. MEGA5: molecular evolutionary genetics analysis using maximum likelihood, evolutionary distance, and maximum parsimony methods. Mol. Biol. Evol. 28, 2731-2739.

Uhlig, S., Busman, M., Shane, D.S., Rønning, H., Rise, F., Proctor, R.H., 2012. Identification of early fumonisin biosynthetic intermediates by inactivation of the FUM6 gene in Fusarium verticillioides. J. Agric. Food Chem. 60, 10293-10301.

Varga, J., Kevei, F., Hamari, Z., Tóth, B., Téren, J., Croft, J.H., Kozakiewicz, Z 2000. Genotypic and phenotypic variability among black aspergilli. In: Samson, R.A., Pitt, J.I. (Eds.), Integration of Modern Taxonomic Methods for Penicillium and Aspergillus Classification. Harwood Academic Publishers, Amsterdam, pp. 397411.

Varga, J., Kocsubé, S., Suri, K., Szigeti, G., Szekeres, A., Varga, M., Tóth, B., Bartók, T., 2010. Fumonisin contamination and fumonisin producing black Aspergilli in dried vinefruits of different origin. Int. J. Food Microbiol. 143, 143-149.
Varga, J., Frisvad, J.C., Kocsubé, S., Brankovics, B., Tóth, B., Szigeti, G., Samson, R.A., 2011. New and revisited species in Aspergillus section Nigri. Stud. Mycol. 69, 117.

Ward, T.J., Bielawski, J.P., Kistler, H.C., Sullivan, E., O’Donnell, K., 2002. Ancestral polymorphism and adaptive evolution in the trichothecene mycotoxin gene cluster of phytopathogenic Fusarium. Proc. Natl. Acad. Sci. USA 99, 9278-9283.

Wicklow, D.T., Dowd, P.F., Alftafta, A.A., Gloer, J.B., 1996. Ochratoxin A: an antiinsectan metabolite from the sclerotia of Aspergillus carbonarius NRRL 369. Can. J. Microbiol. 42, 1100-1103.

Wiemann, P., Sieber, C.M.K., von Bargen, K.W., Studt, L., Niehaus, E.M., Espino, J.J. Huß, K., Michielse, C.B., Albermann, S., Wagner, D., Bergner, S.V., Connolly, L.R. Fischer, A., Reuter, G., Kleigrewe, K., Bald, T., Wingfield, B.D., Ophir, R., Freeman, S., Hippler, M., Smith, K.M., Brown, D.W., Proctor, R.H., Münsterkötter, M., Freitag, M., Humpf, H.-U., Güldener, U., Tudzynski, B., 2013. Deciphering the cryptic genome: genome-wide analyses of the rice pathogen Fusarium fujikuroi reveal complex regulation of secondary metabolism and novel metabolites. PLOS Path. 9, 1-35.

Woloshuk, C.P., Shim, W.B., 2013. Aflatoxins, fumonisins, and trichothecenes: a convergence of knowledge. FEMS Microbiol. Rev. 37, 94-109.

Zaleta-Rivera, K., Xu, C., Yu, F., Butchko, R.A.E., Proctor, R.H., Hidalgo-Lara, M.E., Raza, A., Dussault, P.H., Du, L., 2006. A bidomain nonribosomal peptide synthetase encoded by FUM14 catalyzes the formation of tricarballylic esters in the biosynthesis of fumonisins. Biochemistry 45, 2561-2569. 